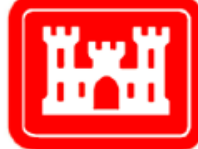

US Army Corps

of Engineers ${ }_{\circledast}$

Engineer Research and

Development Center

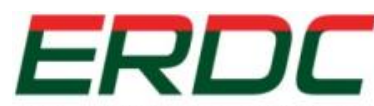

INNOVATIVE SOLUTIONS for a safer, better world

ERDC 6.2 Geospatial Research and Engineering (GRE) ARTEMIS STO-R DUST-CLOUD Subjective Mapping of Dust-Emission Sources by Using MODIS Imagery

Reproducibility Assessment

Samantha N. Sinclair and Sandra L. Jones

May 2017

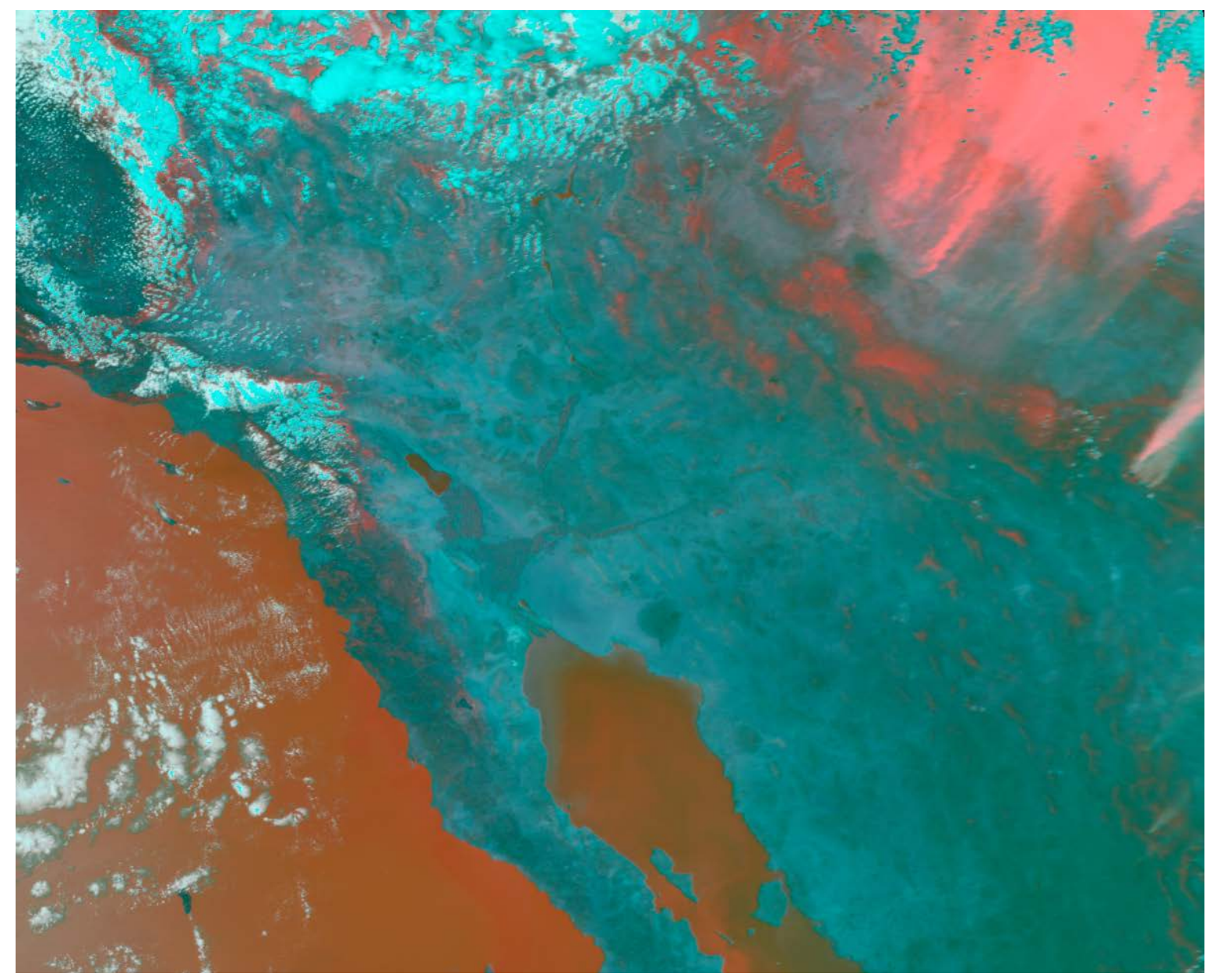


The U.S. Army Engineer Research and Development Center (ERDC) solves the nation's toughest engineering and environmental challenges. ERDC develops innovative solutions in civil and military engineering, geospatial sciences, water resources, and environmental sciences for the Army, the Department of Defense, civilian agencies, and our nation's public good. Find out more at www.erdc.usace.army.mil.

To search for other technical reports published by ERDC, visit the ERDC online library at http://acwc.sdp.sirsi.net/client/default. 


\section{Subjective Mapping of Dust-Emission Sources by Using MODIS Imagery}

\section{Reproducibility Assessment}

Samantha N. Sinclair and Sandra L. Jones

U.S. Army Engineer Research and Development Center (ERDC)

Cold Regions Research and Engineering Laboratory (CRREL)

72 Lyme Road

Hanover, NH 03755-1290

Final Report

Approved for public release; distribution is unlimited.

Prepared for Assistant Secretary of the Army for Acquisition, Logistics, and Technology 103 Army Pentagon

Washington, DC 20314-1000

Under ERDC 6.2 Geospatial Research and Engineering (GRE) Army Terrestrial-Environmental Modeling and Intelligence System Science Technology ObjectiveResearch (ARTEMIS STO-R), "Dynamic Undisturbed Soils Testbed to Characterize Local Origins and Uncertainties of Dust (DUST-CLOUD)" 


\section{Abstract}

Dust storms (5 to $100 \mathrm{~km}$ across) often originate from multiple dust-emission sources (1 to $10 \mathrm{~km}$ across). Remote-sensing-based dust-source identification is a challenge. A previous study developed a subjective approach for mapping dust sources by using enhanced MODIS satellite imagery; therefore, this study conducted mapping exercises to assess the reproducibility of this technique amongst multiple analysts and in different regions. Multiple staff members independently analyzed satellite imagery for mappable dust sources for Southwest Asia and the Southwest United States. Mapped points were considered reproducible if the location of dust emission plumes identified by all participants could be constrained to a $10 \mathrm{~km}$ buffer. Using this definition, point-source locations were $28 \%$ reproducible in Southwest Asia and 85\% reproducible in the Southwest United States. Increasing the allotted buffer to $15 \mathrm{~km}$, however, improved results to $71 \%$ and $100 \%$, respectively. Mapped dust sources for Southwest Asia were compared to geomorphic landform maps. At the 1:750,000 map scale, points mapped by all analysts for a single dust plume tended to overlay one landform, while at the 1:100,000 map scale, points were strewn across several landforms. Results suggest that the methodology is reproducible for certain applications but that location-uncertainty tolerance affects perceived conclusions.

DISCLAIMER: The contents of this report are not to be used for advertising, publication, or promotional purposes. Citation of trade names does not constitute an official endorsement or approval of the use of such commercial products. All product names and trademarks cited are the property of their respective owners. The findings of this report are not to be construed as an official Department of the Army position unless so designated by other authorized documents.

DESTROY THIS REPORT WHEN NO LONGER NEEDED. DO NOT RETURN IT TO THE ORIGINATOR. 


\section{Contents}

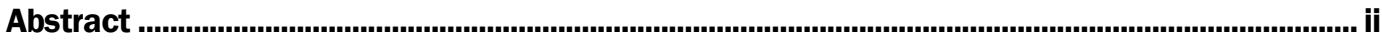

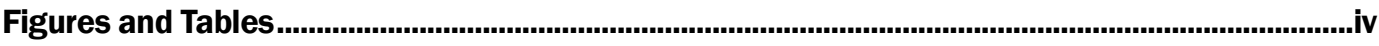

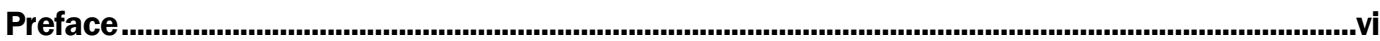

Acronyms and Abbreviations ..........................................................................................................vii

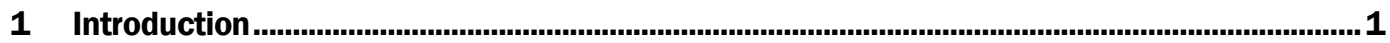

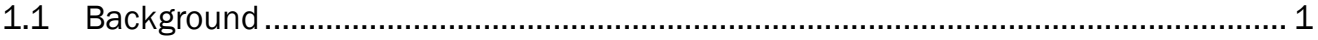

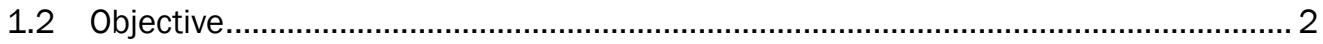

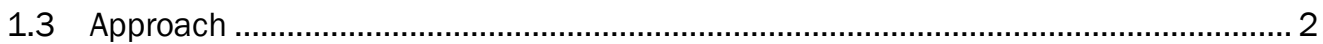

2 Methods ..................................................................................................................................... 3

2.1 Satellite imagery acquisition and selection process ............................................. 4

2.2 Mapping dust-plume-head point sources......................................................... 8

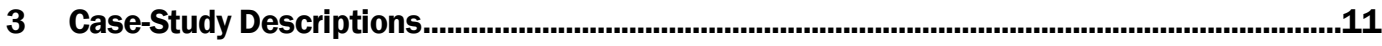

3.1 Southwest Asia .................................................................................................. 11

3.2 Southwest United States ............................................................................... 12

4 Results ................................................................................................................................14

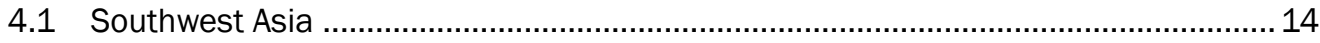

4.2 Southwest United States ............................................................................. 17

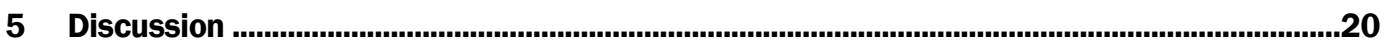

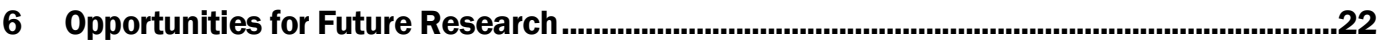

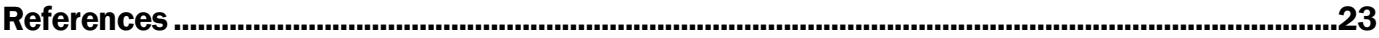

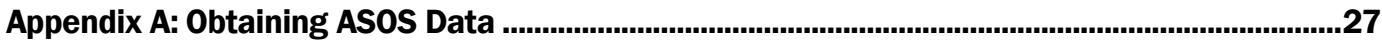

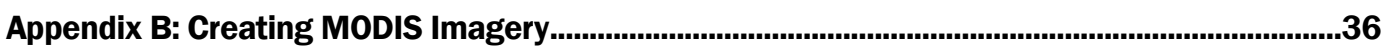

Appendix C: ArcMap Mapping Procedures.......................................................................................49

Report Documentation Page 


\section{Figures and Tables}

\section{Figures}

1 MODIS satellite imagery of the Southwest United States and Mexico. Panels $A, B$, and $C$ depict the area outlined by the red box within the inset of Panel $A$. Panel $A$ is a True Color image that depicts white clouds against a brown landscape; a bird's eye representation of Earth. Panel $B$ is a MILLER image that depicts aqua colored clouds against a dark blue/green landscape. Lofted dust appears pink while high-relief topography appears red. The white dashed box depicts a region that does not contain any lofted dust. Panel $C$ is a EUMETSAT image that depicts red clouds against a purple landscape. Lofted dust appears hot pink. The white circle in each panel depicts a dust event. Note the pink dust that can be seen in Panels $B$ and $C$ is undetectable in Panel $A$.

2 True Color (left) and MILLER (right) images depicting a dust event on the Colorado Plateau in northeast Arizona in the area outlined by the red box. Note that while there is a considerable amount of pink-colored portions of the MILLER image, discernable plume-head point sources are not apparent and therefore cannot be deduced or mapped

3 True Color (top left), MILLER (top right), and EUMETSAT (bottom left) images for the region outlined by the red box over portions of the Southwest United States and Mexico. These images highlight the potential for high-relief terrain features to be misinterpreted as dust. Note the light pink colored feature to the east of the Gulf of California in the MILLER image that resembles the appearance of lofted dust. When compared to the True Color image, it is apparent that these features are clouds. The white circle in the MILLER image highlights high-relief terrain that has the appearance of highly concentrated lofted dust. This same region outlined in the EUMETSAT image, however, does not show any indication of lofted dust. The True Color image reveals this region is composed of mountainous terrain

$4 \quad$ As in Fig. 3 but for smoke. True Color (top left), MILLER (top right), and EUMETSAT (bottom left) images for the region outlined by the red box over portions of the Southwest United States and Mexico. The MILLAR and EUMETSAT images reveal a mesoscale dust event in the northeast corner of the domain. A single plume located south of the dust storm also looks as if it could be lofted dust. This same feature is apparent in the True Color image but very clearly originates from forested terrain, indicating that it is very likely smoke from a forest fire.

5 Map of Southwest Asia (as outlined by the red box) depicting western India, Pakistan, Iran, Afghanistan, Oman, the United Arab Emirates, and eastern Saudi Arabia. The white box shows the location of the Hamun dry lakes, which are an important dust source in the region. The enlarged image box in the top right depicts the individual lakebed boundaries (modified from Rashki et al. 2013). Dust is lofted from the Hamun region and transported in a southeasterly direction around the Chagai Hills and into Pakistan

6 Map of the Southwest United States desert regions. The boundaries of the four major deserts in this region, including the Great Basin, Mojave Desert, Sonoran Desert, and Chihuahuan Desert, are outlined (modified from EPA 2012) 13

$7 \quad$ MILLER image (top) depicting a dust event on 16 September 2011 in Southwest Asia and associated dust-plume-head markers mapped by five analysts. The 
region in the white rectangle is enlarged in the bottom panels. The 1:750,000 (bottom left) and 1:100,000 (bottom right) scale geomorphic landform maps are overlain with mapped plume-head point sources. The oval in each panel outlines five points placed by each analyst for single plume head

8 Quality scores assigned by each of the five analysts to points mapped in the Southwest Asia case study. Points marked with high confidence (3) are green, moderate confidence (2) are yellow, and low confidence (1) are red. Each panel depicts the points placed by an individual analyst. Note that points mapped by all analysts were assigned a higher quality score (and were therefore easier to identify and map) than those mapped by only one or two analysts.

9 MILLER image (left) depicting a dust event on 2 April 2003 in the Southwest United States and the associated dust-plume head markers mapped by four analysts. Points mapped by each analyst are represented by different colored circles. Note the five points mapped by analyst 1 in the southeast corner of the image that are incorrectly placed on clouds. The area outlined in the black rectangle is enlarged on the right: a close-up of individual plume-head point sources mapped by each analyst. Also, note the variability in the number of plume heads subjectively identified by each analyst. Buffers placed around plume heads mapped by all analysts show a high degree of duplicability and are constrained to $3 \mathrm{~km}$ (blue circle), $5 \mathrm{~km}$ (green circles), and $10 \mathrm{~km}$ (yellow circles) buffers. This image does not include the $25 \mathrm{~km}$ buffer

10 Quality scores assigned by each of the four analysts to points mapped in the Southwest United States case study (shown in Fig. 9). Points marked with high confidence (3) are green, moderate confidence (2) are yellow, and low confidence (1) are red. Each panel depicts the points placed by an individual analyst. As with the Southwest Asia study, plume heads marked by all analysts received a higher quality score than those mapped by just one or two analysts.

\section{Tables}

1 MODIS channels used to generate case-study imagery

2 Number of mapped dust sources and mean quality scores for the Southwest Asia 16 September 20110650 UTC case study

3 Number of mapped dust sources and mean quality scores for the Southwest United States 2 December 20032050 UTC case study. 


\section{Preface}

This study was conducted for the Assistant Secretary of the Army for Acquisition, Logistics, and Technology under the U.S. Army Engineer Research and Development Center (ERDC) 6.2 Geospatial Research and Engineering (GRE) Program's Army Terrestrial-Environmental Modeling and Intelligence System Science Technology Objective--Research (ARTEMIS STO-R), "Dynamic Undisturbed Soils Testbed to Characterize Local Origins and Uncertainties of Dust (DUST-CLOUD)." The technical monitor was Ms. Sandra L. J ones, ERDC Cold Regions Research and Engineering Laboratory (CRREL).

The work was performed by the Terrestrial and Cryospheric Sciences Branch (CEERD-RRG) of the Research and Engineering Division (CEERD-RR), ERDC-CRREL. At the time of publication, CDR J. D. Horne, USN (Ret), was Chief, CEERD-RRG and CEERD-RR. The Deputy Director of ERDC-CRREL was Dr. Lance Hansen and the Director was Dr. J oseph L. Corriveau.

COL Bryan S. Green was the Commander of ERDC, and Dr. David W. Pittman was the Director. 


\section{Acronyms and Abbreviations}

ARTEMIS STO-R Army Terrestrial-Environmental Modeling and Intelligence System Science Technology Objective-Research

ASOS

Automated Surface Observing System

COAMPS

Coupled Ocean-Atmosphere Mesosphere Prediction System

CRREL

Cold Regions Research and Engineering Laboratory

DUST-CLOUD

Dynamic Undisturbed Soils Testbed to Characterize Local Origins and Uncertainties of Dust

ERDC

U.S. Army Engineer Research and Development Center

EUMETSAT

European Organization for the Exploitation of Meteorological Satellites

GIS

Geospatial Information System

GRE

Geospatial Research and Engineering

HDF

Hierarchical Data Format

MODIS

Moderate Resolution Imaging Spectroradiometer

NASA

National Aeronautics and Space Administration

TIFF

Tagged Image File Format

UTC

Coordinated Universal Time

VIIRS

Visible Infrared Imager Radiometer Suite

WGS

World Geodetic System 



\section{Introduction}

\subsection{Background}

Atmospheric soil and mineral dust can significantly influence a variety of global-scale processes, such as the radiation budget of the atmosphere, biogeochemical reactions that occur in the ocean, and global climate (Shinn et al. 2000; Mahowald et al. 2005, 2014; Ravi et al. 2011; Webb et al. 2012; Huang et al. 2014; Knippertz and Stuut 2014; Skiles et al. 2015; Wang et al. 2017). On regional and local scales, lofted dust can negatively affect visibility, mobility, communication, and human health (Rushing et al. 2005; Rushing and Tingle 2006; De Longueville et al. 2010; Okin et al. 2011; AlHemoud et al. 2017; Middleton 2017;). As a result, understanding the processes that control spatial and temporal patterns of atmospheric dust occurrence has become a priority for the research, military, operational forecasting, and hazard mitigation communities (e.g., Knippertz and Stuut 2014; Sprigg et al. 2014; Shepherd et al. 2016).

Accurate dust-source characterizations are critical for effectively modeling dust storms and their associated hazards. Numerous remote-sensing studies have attempted to identify dust-emission-source locations (e.g., Legrand et al. 1994; Torres et al. 1998; Prospero et al. 2002; Miller 2003; Bullard et al. 2008; Baddock et al. 2009; Walker et al. 2009; Ginoux et al. 2012; J afari and Malekian 2015; Moridnejad et al. 2015; Zhang et al., forthcoming). Historically, satellite-based approaches have struggled with dust-plume origin detection. This is because synoptic and mesoscale dust storms on the order of 5 to $100 \mathrm{~km}$ rarely originate from a single source but rather from the amalgamation of several point-source dust plumes. These smaller plumes stem from multiple point sources (i.e., plume heads 1 to $10 \mathrm{~km}$ across) that can evade coarser-resolution satellite detection.

Advances in satellite image resolution ( $1 \mathrm{~km}$ or better) have greatly enhanced the ability to detect plume heads remotely (Walker et al. 2009; Muhs et al. 2014). Walker et al. (2009) developed an approach to manually map plume-head point sources in a geospatial information system (GIS) framework using Moderate Resolution Imaging Spectroradiometer (MODIS) imagery processed through a dust-enhancement algorithm. With this technique, a location is digitized and archived if the analyst observes an unobscured plume head in the imagery. Although analysts are able to 
map dust sources at the satellite-image grid scale, location analysis error is almost unavoidable. Airborne dust must be sufficiently elevated for overland dust enhancement algorithms to work; otherwise, thermal and reflectance contrast signals between the dust and the underlying surface may not be discernable. By the time the dust has reached a sufficient altitude, it is likely to have traveled downstream from its deflating source. As such, errors in digitized source location may be on the order of 1 to $10 \mathrm{~km}$ (Walker et al. 2009).

Even with this inherent uncertainty, the Walker et al. (2009) technique is a popular approach for dust-source identification and is often used as a proxy for dust-source observations (e.g., Lary et al. 2016). The Walker et al. (2009) methodology is also currently being used to incorporate dust sources into the Navy's Coupled Ocean-Atmosphere Mesosphere Prediction System (COAMPS; e.g., Liu et al. 2007; Walker et al. 2009), which to date remains the highest-resolution dust-source treatment in an operational military weather forecast model. To our knowledge, however, the sensitivity of the Walker et al. (2009) methodology to analyst subjectivity has never been formally assessed.

\subsection{Objective}

The goal of this study is to examine the role of analyst subjectivity on the reproducibility of the Walker et al. (2009) technique.

\subsection{Approach}

A group of analysts independently mapped plume-head point sources for two predetermined case-study dust events, one in the Southwest United States and one in Southwest Asia. Section 2 of this report provides stepby-step instructions for an adapted version of the Walker et al. (2009) methodology and outlines the procedures and criteria used to determine if dust was present in a satellite image. It also includes the GIS mapping procedures. Section 3 outlines the two geographical regions and provides background information pertinent to each case study. Section 4 reviews the resultant plume-head point-source maps. Section 5 discusses final reproducibility assessments and limitations of the approach, and Section 6 identifies potential areas of future work. 


\section{Methods}

For each case-study region, a single analyst downloaded Automated Surface Observing System (ASOS) weather data to identify dusty days reported in local weather station records. The same analyst then downloaded and processed true-color and dust-enhanced MODIS satellite imagery associated with each day of interest and selected a single time period for each regional case study based on a perceived potential for numerous mappable plume-head point sources. After reviewing mapping procedures and techniques, a group of additional analysts then individually mapped plume-head point sources in the images selected for each region by using ArcMap 10.3 and assigned a quality score (confidence level) to each mapped point. Results from each analyst were subsequently aggregated together and assessed for reproducibility.

Because of a general lack of in situ dust-emission observations, there is no way to definitively corroborate the precise locations of historically emitting dust sources on a regional scale or to verify that point sources subjectively identified by an analyst are actually real sources. Thus, quantitative assessment is limited in nature to point-placement reproducibility amongst analysts. In other words, this report addresses the sensitivity of plume-head location placement to analyst interpretation. It does not attempt to quantify the ability of individual group members to reproduce actual emissionsource patterns. As a result, the reproducibility assessments in this report are based only on the plume heads that were identified by all participating analysts. Of those points, a mapped plume-head point source is considered reproducible if all point-source markers are constrained to a $10 \mathrm{~km}$ buffer, a limit defined by the potential for advection error when using the Walker et al. (2009) methodology.

In addition to mapping plume-head point sources, an overlay analysis using geomorphic landform maps was also conducted for the Southwest Asia case study. Certain arid-region landform types tend to be richer in dustsized material than others (e.g., Bullard et al. 2011; Sweeney et al. 2011, 2016; Sweeney and Mason 2013) because processes that dictate landform evolution also influence soil development. As a result, some researchers believe that landform designations can be used as a suitable proxy for soil attributes like dust-emission potential (e.g., Bullard et al. 2008; Bacon and McDonald 2016). For example, dry lakebeds filled with unconsolidated 
fine-grained sediments are commonly considered a significant dust-emission source globally (e.g., Prospero et al. 2002). Because of this important link between landform type and dust emissions, a more comprehensive understanding of the geomorphic controls that dictate atmospheric dust is necessary to enhance weather and climate models (Baddock et al. 2016). Thus, an overlay analysis may offer insight as to whether the Walker et al. (2009) technique may be a reliable option for validating various geomorphic-based dust-source characterization approaches.

\subsection{Satellite imagery acquisition and selection process}

All satellite images used in this study were derived from MODIS data, which have been collected by the Earth Observing System Terra and Aqua satellites since early 2000 and mid-2002, respectively (see https://modis.gsfc.nasa.gov/). ASOS weather station data ranging from $1 \mathrm{~J}$ anuary 2000 to $30 \mathrm{~J}$ une 2016 was downloaded for the case-study regions and inspected for accounts of airborne dust to help select appropriate satellite imagery for the reproducibility experiments. These ASOS stations record sub-hourly meteorological data and are found primarily at airports across the globe (Nadolski 1998). Appendix A provides step-by-step instructions for ASOS data acquisition.

Periods of ASOS station records with entries of "blowing dust," "dust devils," or "sandstorm" in the obscuration-to-vision column (i.e., the "obscure" column) were noted for consideration. These data, however, required further reduction as not all dust events identified via ground-based sensors are detectable by satellites as previously discussed. Visibility observations greater than approximately $32 \mathrm{~km}$ were used to filter out isolated events or dust events likely undetectable by the MODIS sensors. Viable case-study options were further reduced to periods with several (five to ten) weather stations simultaneously reporting dust or stations reporting long periods (hours to days) of near continuous blowing dust, as these are strong indicators of widespread dust lofting through synoptic or mesoscale forcing mechanisms.

For each potential period identified via the ASOS record inspection, a single analyst downloaded MODIS Level 1B $1 \mathrm{~km}$ Calibrated Radiance granules. These granules were originally downloaded as HDF (hierarchical data format) files, post-processed, and reformatted into georeferenced TIFF (tagged image file format) imagery that could be directly imported into ArcGIS. Three unique images were then generated from each acquired 
granule (e.g., Figure 1) using the channels listed in Table 1 and a script developed in Python (see Appendix B). The first is a true-color image, a bird's eye representation of the Earth from space without the addition of any filtering or post processing (e.g., Figure 1a). The other two images were created using false-color dust-enhancement algorithms (e.g., Figures $1 \mathrm{~b}$ and $1 \mathrm{c})$.

The first dust-enhanced image is rendered using a technique by Miller (2003), which uses visible, near-infrared, thermal-infrared, and water-vapor channels to distinguish elevated dust from the underlying background. The Miller (2003) approach uses different equations to set the resulting red color over land and water. Lofted dust appears pink, landscapes have blue and green hues, water and steep terrain are red, and clouds appear aqua or cyan (see Figure 1b). For a detailed overview of the algorithm procedure, see section 3 of Miller (2003).

The second dust-enhanced image is generated using an algorithm originally developed by the European Organization for the Exploitation of $\mathrm{Me}$ teorological Satellites (EUMETSAT) for use with their geostationary satellite data. This algorithm has since been adapted for other satellite platforms (e.g., Lensky and Rosenfeld 2008; Brindley et al. 2012). Unlike the Miller (2003) approach, the EUMETSAT technique does not require use of any visible channels, making it useful for both day and nighttime dust detection. Lofted dust often appears hot pink against a purple landscape below, and thick clouds typically appear red, which can camouflage the pink dust in some instances (see Figure 1c). An overview of the EUMETSAT algorithm and procedures for setting imagery color palettes are available in section 2 of Brindley et al. (2012), and step-by-step instructions to render MODIS imagery are located at the end of this report in Appendix B. Imagery created with true-color settings, the Miller (2003) algorithm, and the EUMETSAT algorithm are labeled "True Color," "MILLER," and "EUMETSET," respectively, throughout this report. 
Figure 1. MODIS satellite imagery of the Southwest United States and Mexico. Panels $A, B$, and $C$ depict the area outlined by the red box within the inset of Pane/ $A$. Panel $A$ is a True Color image that depicts white clouds against a brown landscape; a bird's eye representation of Earth. Panel $B$ is a MILLER image that depicts aqua colored clouds against a dark blue/green landscape. Lofted dust appears pink while high-relief topography appears red. The white dashed box depicts a region that does not contain any lofted dust. Panel $C$ is a EUMETSAT image that depicts red clouds against a purple landscape. Lofted dust appears hot pink. The white circle in each panel depicts a dust event. Note the pink dust that can be seen in Panels $B$ and $C$ is undetectable in Panel $A$.
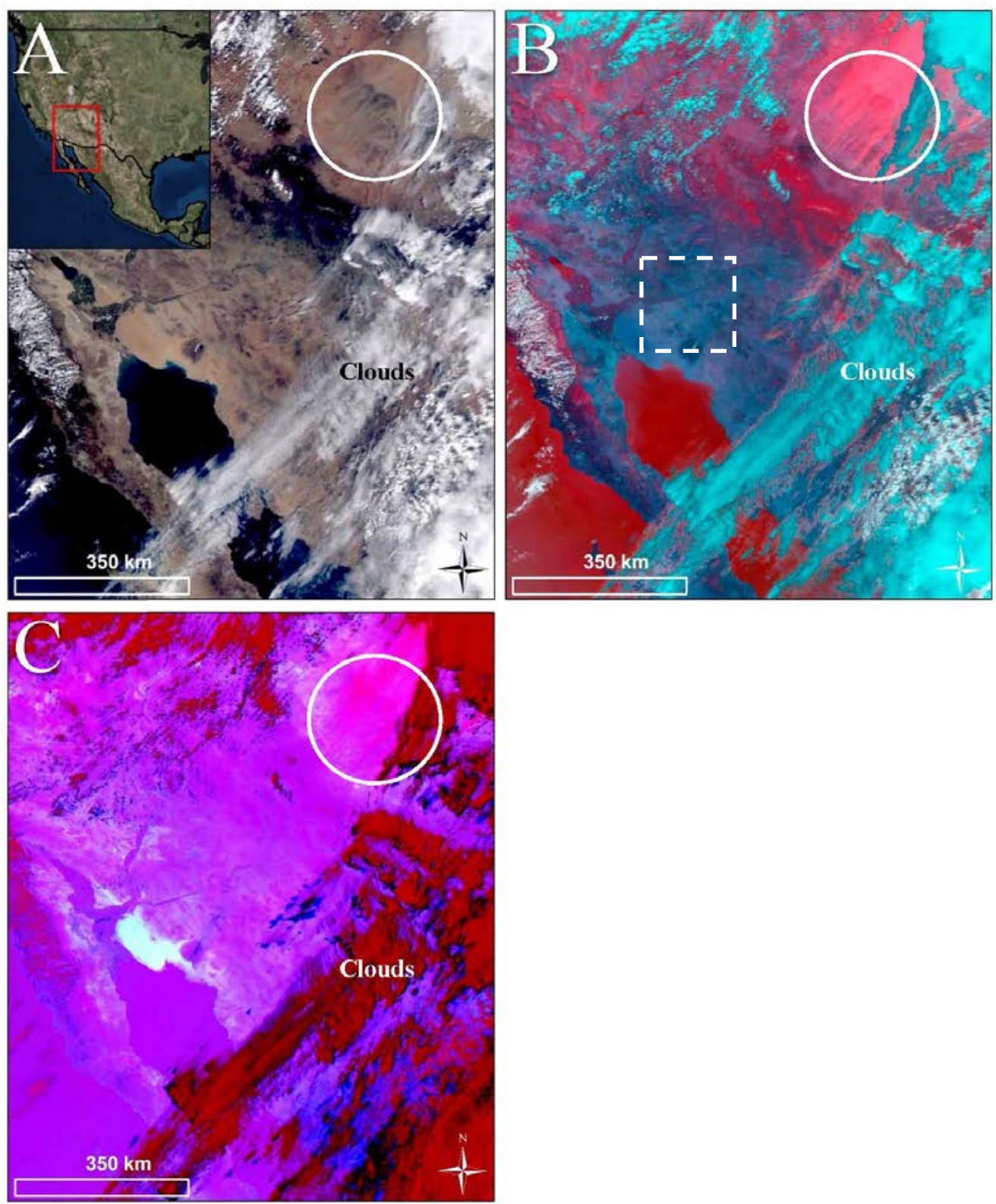
Table 1. MODIS channels used to generate case-study imagery.

\begin{tabular}{|c|c|c|}
\hline MODIS Channel & Description & Band Width $(\mu \mathrm{m})$ \\
\hline 1 & Red & $0.620-0.670$ \\
\hline 2 & Near Infrared & $0.841-0.876$ \\
\hline 3 & Blue & $0.459-0.479$ \\
\hline 4 & Green & $0.545-0.565$ \\
\hline 26 & Water Vapor & $1.360-1.390$ \\
\hline 29 & Water Vapor & $8.400-8.700$ \\
\hline 31 & Infrared & $10.780-11.280$ \\
\hline 32 & Infrared & $11.770-12.270$ \\
\hline
\end{tabular}

Of all the MODIS images that were downloaded for this study, only a select few were useful for mapping plume-head point sources. This was because of a variety of reasons, including but not limited to the Terra and Aqua satellites not passing over the area of interest during the time of the dust storm; the presence of cloud coverage obscuring the dust below; or the occurrence of a low-concentration, short-lived, insufficiently lofted, or nighttime dust storm. In addition, analysts were not able to map lofted dust in all imagery that coincided with an event as, in many instances, dust appeared as an effusive cloud without discernable point sources that could identified or mapped (Figure 2).

Figure 2. True Color (left) and MILLER (right) images depicting a dust event on the Colorado Plateau in northeast Arizona in the area outlined by the red box. Note that while there is a considerable amount of pink-colored portions of the MILLER image, discernable plume-head point sources are not apparent and therefore cannot be deduced or mapped.
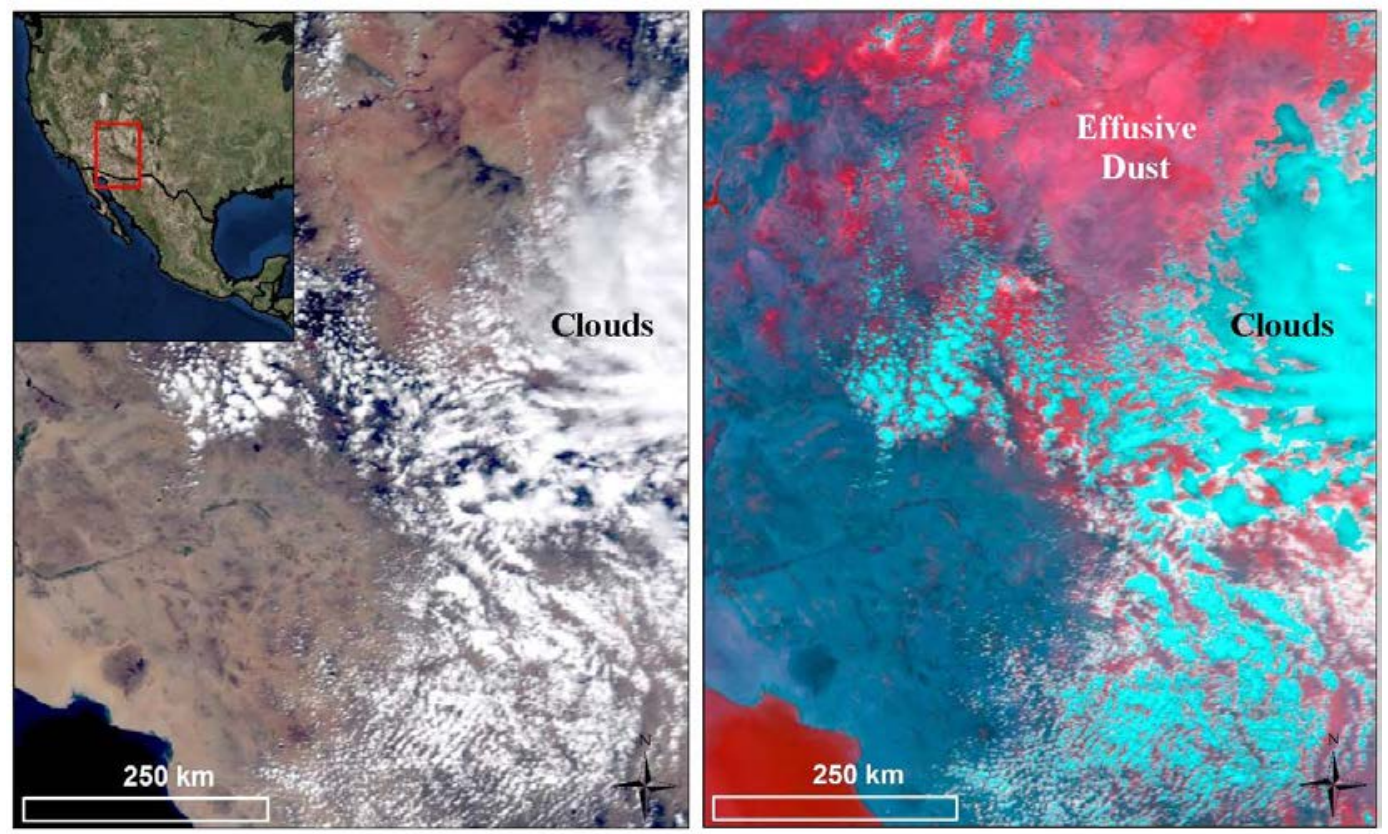
Other images contained plume-head-like features that initially looked like mappable sources; but further inspection identified them as thin clouds, forest fires, or high-relief ridges. Clouds often looked deceivingly like lofted dust in the MILLER images, particularly when relatively thin (e.g., Figure 3). To ensure that the team did not mistakenly map clouds as dust, analysts used the True Color and EUMETSAT images to clearly define the location of any clouds in the region before mapping plume-head point sources. Large-scale forest fires and certain landforms also portrayed dustlike characteristics in the MILLER images. Some high-relief landforms, such as narrow mountain ridges, appeared reddish/ pink in the images, mimicking the appearance of a long dust-plume tail (see Figure 3). Smoke from forest fires appeared as a plume-like feature that emanated from a single point source and looked nearly identical to plume heads in the MILLER and EUMETSAT images (Figure 4). To ensure that these features were not accidentally mapped as dust sources, the analysts used the True Color image to evaluate where the dust sources were coming from off the landscape. Narrow mountain ridges were easily identifiable in the True Color images and could immediately be ruled out, and smoke typically originated in forested terrains that were very unlikely to be large dust producers.

\subsection{Mapping dust-plume-head point sources}

Once a single set of the True Color and dust-enhanced images was selected from the remaining list of options for each case-study region, the analysts mapped plume heads using ArcMap 10.3 software. This involved uploading the processed MODIS imagery and creating and editing shapefiles of point markers for each region. All participating analysts then independently mapped point sources they identified through subjective imagery interpretation. Each analyst also assigned a quality score to every mapped point as a means to communicate user confidence in plume-head mapping decisions. Quality scores were ranked on a qualitative scale from 1 to 3. Users assigned a particular point a quality score of 3 only when they were confident in the placement of the point and felt certain dust was originating from that location. A quality score of 2 meant users were almost certain dust was coming from that location but were not entirely confident. A quality score of 1 meant the user was not certain in the placement of the point. Appendix C outlines the detailed step-by-step instructions for the mapping procedures and quality score assignment followed in ArcMap. 
Figure 3. True Color (top left), MILLER (top right), and EUMETSAT (bottom left) images for the region outlined by the red box over portions of the Southwest United States and Mexico. These images highlight the potential for high-relief terrain features to be misinterpreted as dust. Note the light pink colored feature to the east of the Gulf of California in the MILLER image that resembles the appearance of lofted dust. When compared to the True Color image, it is apparent that these features are clouds. The white circle in the MILLER image highlights high-relief terrain that has the appearance of highly concentrated lofted dust. This same region outlined in the EUMETSAT image, however, does not show any indication of lofted dust. The True Color image reveals this region is composed of mountainous terrain.
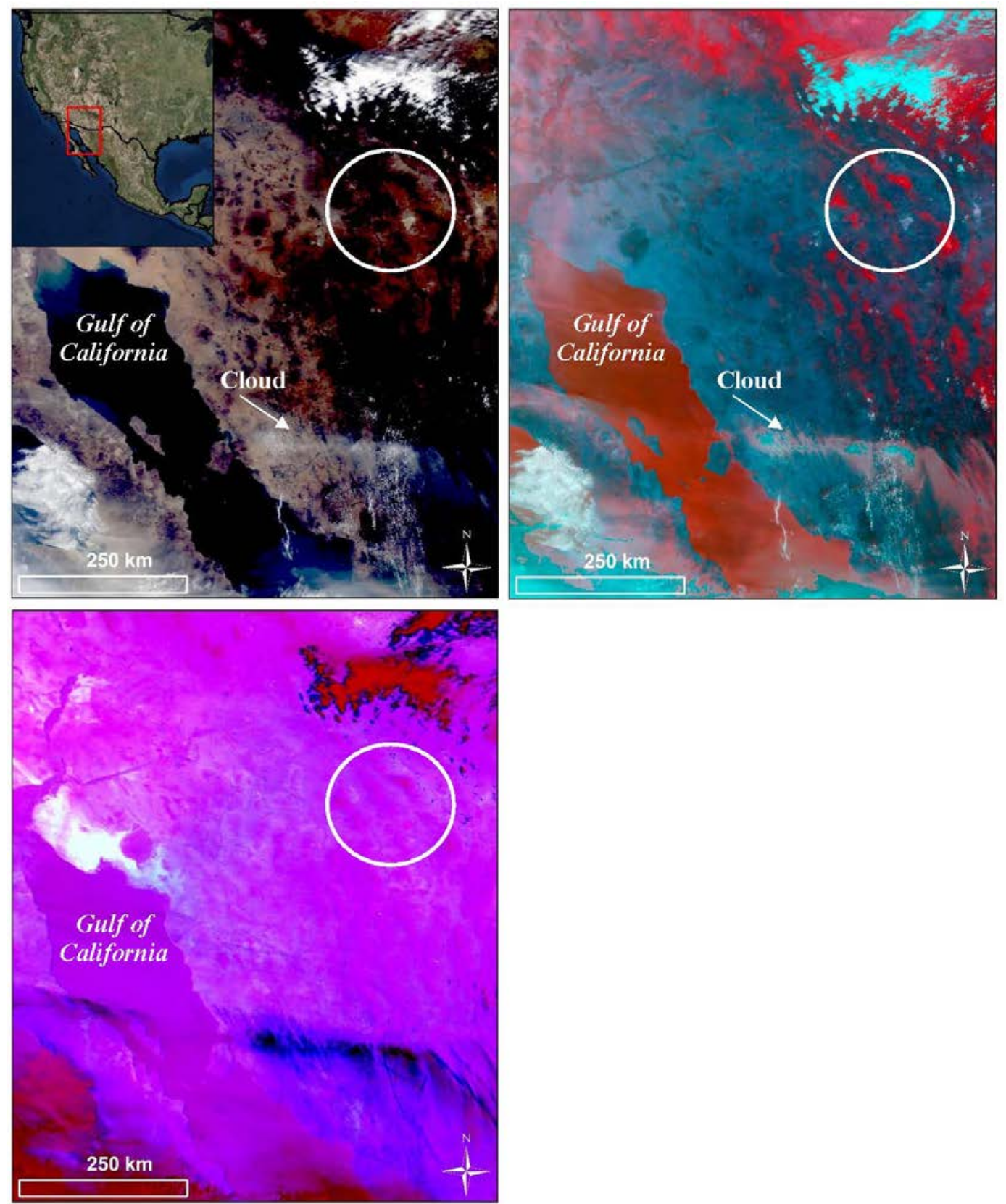
Figure 4. As in Fig. 3 but for smoke. True Color (top left), MILLER (top right), and EUMETSAT (bottom left) images for the region outlined by the red box over portions of the Southwest United States and Mexico. The MILLAR and EUMETSAT images reveal a mesoscale dust event in the northeast corner of the domain. A single plume located south of the dust storm also looks as if it could be lofted dust. This same feature is apparent in the True Color image but very clearly originates from forested terrain, indicating that it is very likely smoke from a forest fire.
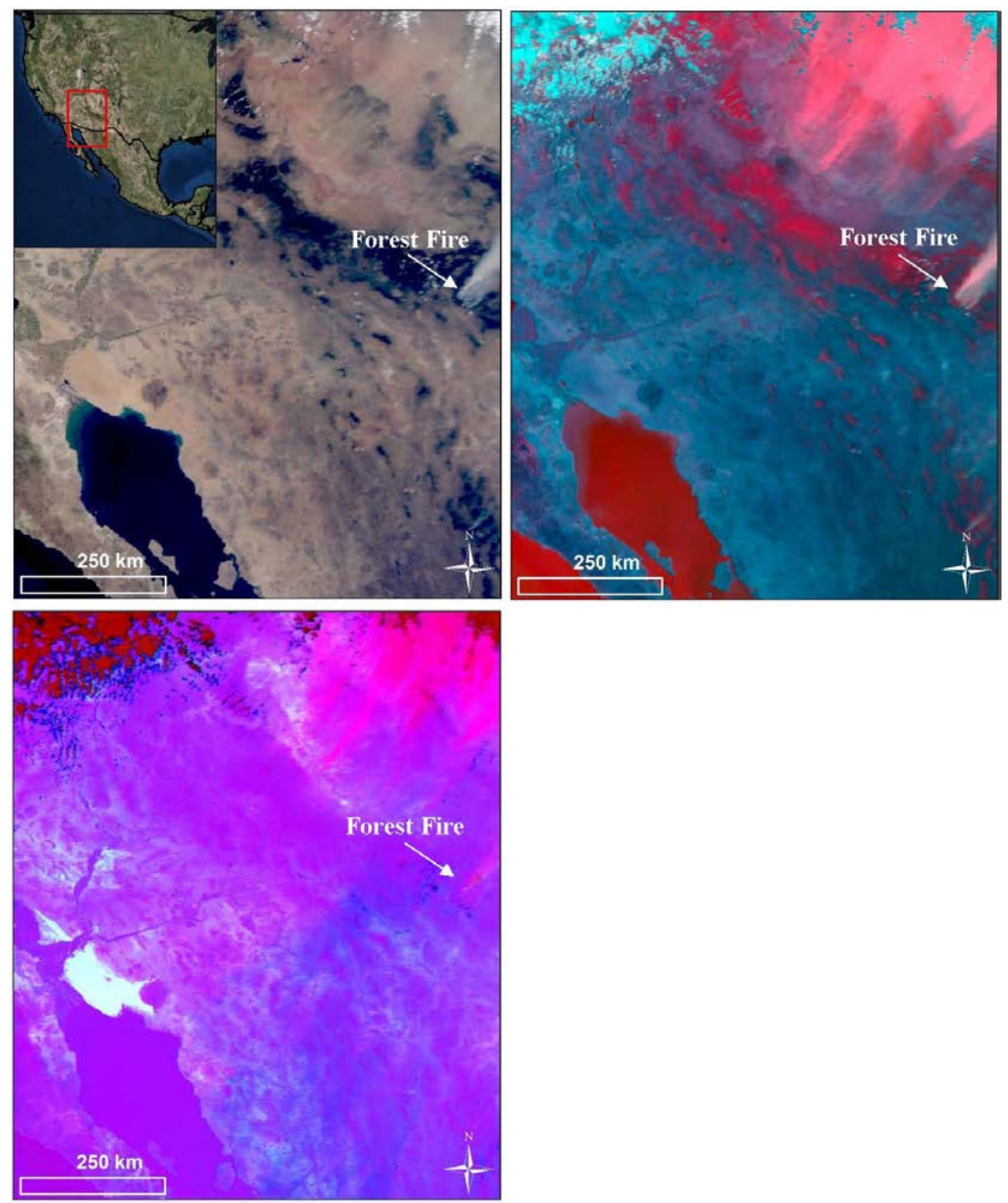


\section{Case-Study Descriptions}

\subsection{Southwest Asia}

The desert region of Southwest Asia is composed of several countries, including Saudi Arabia, Oman, the United Arab Emirates, Iran, Afghanistan, Pakistan, and India (Figure 5). The geomorphology of this region is highly variable and includes mountainous terrain, river valleys, plateaus, dry washes, ephemeral lakes, alluvial fans, sand dunes, and salt plains (Goudie and Middleton 2006; Affleck et al. 2011). Vegetation is sparse but diverse due to the variety of unique landscapes present. Common types of vegetation include camel brush, broom grass, buckthorn, and a variety of trees in the mountainous regions (Khaurin 2003; Breckle 2007).

Figure 5. Map of Southwest Asia (as outlined by the red box) depicting western India, Pakistan, Iran, Afghanistan, Oman, the United Arab Emirates, and eastern Saudi Arabia. The white box shows the location of the Hamun dry lakes, which are an important dust source in the region. The enlarged image box in the top right depicts the individual lakebed boundaries (modified from Rashki et al. 2013). Dust is lofted from the Hamun region and transported in a southeasterly direction around the Chagai Hills and into Pakistan.

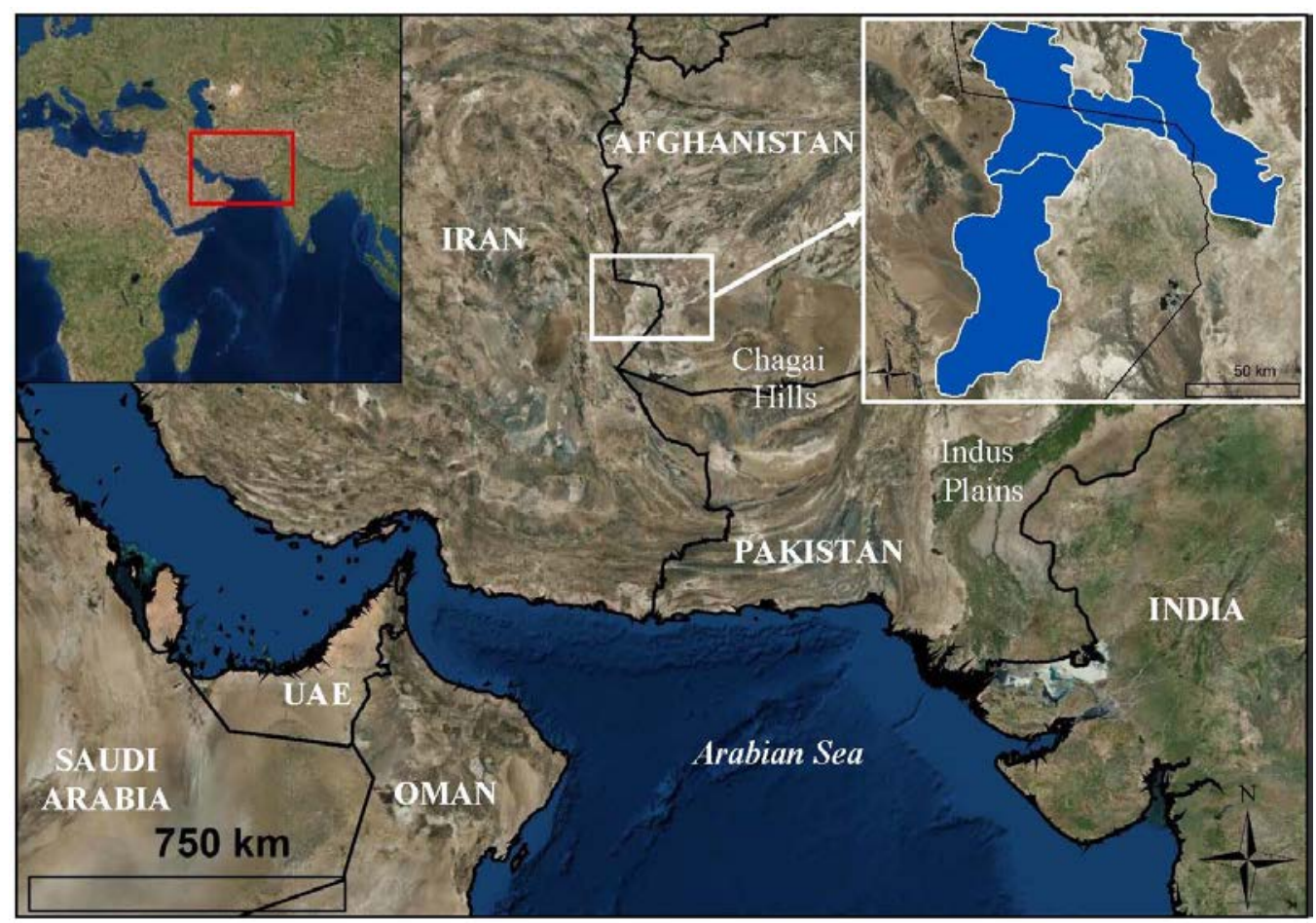

The majority of dust storms in Southwest Asia occur at the convergence between the borders of Iran, Pakistan, and Afghanistan (Goudie and Middleton 2006). Other sources of dust in the region include the coast of the 
Arabian Sea in Iran and the Indus Plains in Pakistan, and dust originating in the Sahara Desert is also frequently transported to the area (see Figure 5) (Goudie and Middleton 2006). At the convergence between Iran, Pakistan, and Afghanistan, dust sources are located in the lowland valleys between mountain peaks. Major dust emitters in the region include alluvial fans and ephemeral lakes, such as the Hamun dry lakes and the deltaic fan associated with the Helmand River. Dense dust plumes often originate from these sources and are transported by strong northerly winds blowing to the southeast through lowland areas and around the Chagai Hills (see Figure 5) (Goudie and Middleton 2006; Muhs et al. 2014). This is a dominant pattern often seen in the area and is representative of the specific dust storm that took place on 16 September 2011 and was mapped in this study. Images were generated from the MODIS Terra 0650 UTC* (1120 local time in Southwest Asia) granule.

\subsection{Southwest United States}

The desert region of the Southwest United States encompasses portions of California, Nevada, Utah, Arizona, New Mexico, and Texas between the Pacific Ocean and the Rocky Mountains and is composed of four major deserts: the Great Basin, Mojave, Sonoran, and Chihuahuan (Figure 6). The regional geomorphology includes wash plains, dry lakebeds, playas, sand dunes, floodplains, alluvial plains, and isolated mountain ranges shaped by erosional processes. Vegetation in the region, though sparse, does not conform to the near-void vegetation patterns typically seen in other desert regions globally. Instead, a denser population of desert shrub, brush, grasses, and cacti, including plants such as cheat grass, creosote, mesquite, atriplex, and ironwood, cover a larger percentage of the surface.

Dust storms occur throughout the year in the Southwest United States (driven by prevailing wind patterns), though seasonal fluctuations in the Pacific High (a semi-permanent, subtropical anticyclone located over the northeastern Pacific Ocean) and the J et Stream drive variations in the number and intensity of dust storms observed throughout the year (Adams and Comrie 1997; Tong et al. 2012). During the monsoon season (J uly to mid-September), dust events are usually induced by localized thunderstorms. The dust storm mapped in this study is associated with a weather front that moved across the Colorado Plateau in Arizona on 2 April 2003.

* Coordinated Universal Time 
Images were generated from the MODIS Terra 2050 UTC (1250 local time in the Southwest United States) granule.

Figure 6. Map of the Southwest United States desert regions. The boundaries of the four major deserts in this region, including the Great Basin, Mojave Desert, Sonoran Desert, and Chihuahuan Desert, are outlined (modified from EPA 2012).

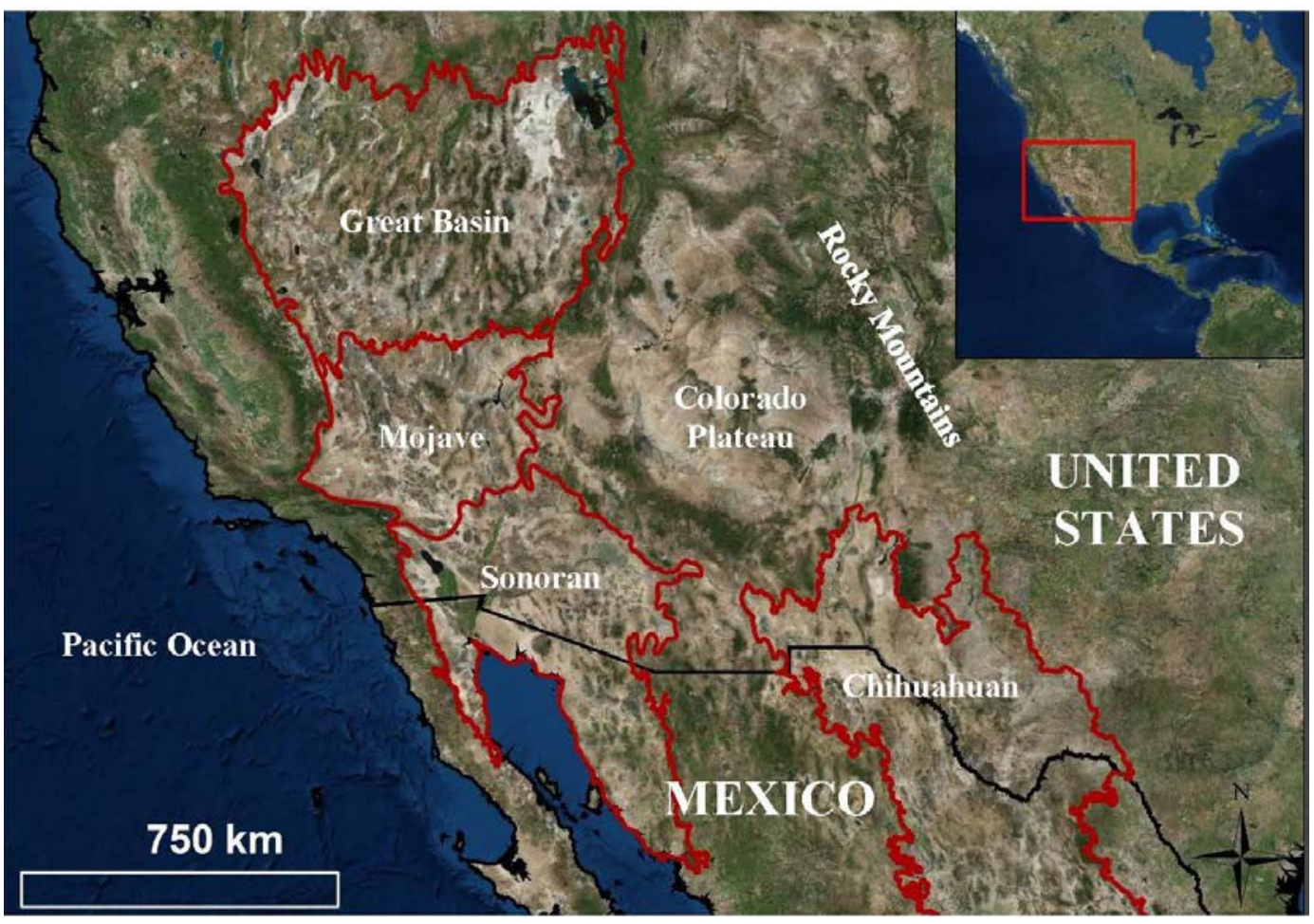




\section{Results}

\subsection{Southwest Asia}

Table 2, Figure 7, and Figure 8 show the results from the reproducibility experiment conducted for Southwest Asia. As shown in Table 2, the number of plume-head point sources mapped by each analyst ranged from 10 to 28 , with an average of $19 \pm 6.9$. Mean quality scores between analysts ranged from 1.94 to 2.5 , with an average of $2.15 \pm 0.22$. The analyst that mapped the greatest number of point sources was second to least confident in their decisions while the analyst that mapped the fewest points was the most confident. In total, there were seven plume heads that all five analysts mapped (see Figures 7 and 8 ). Of these, two plume heads fell within a $10 \mathrm{~km}$ buffer while all others fell within a $15 \mathrm{~km}$ (three plume heads), 25 $\mathrm{km}$ (one plume head), or $35 \mathrm{~km}$ (one plume head) buffer. Other plume heads depicted in the image were only mapped by a portion (four or fewer analysts) of the team and thus were not considered for reproducibility evaluation.

Results were mapped onto a 1:100,000 and a 1:750,000 geomorphic landform map produced by the Desert Research Institute (see McDonald et al. 2013 for landform map generation and attribute details). At a 1:750,000 map scale, plume-head point sources mapped by all five analysts for a single plume head fell within the same type of landform (alluvial plain), while at the 1:100,000 map scale, the same points were strewn across several different landform types (delta plain, playa, wet playa, and sand sheets; see Figure 7).

Table 2. Number of mapped dust sources and mean quality scores for the Southwest Asia 16 September 20110650 UTC case study.

\begin{tabular}{|c|c|c|}
\hline Analyst Identification Number & $\begin{array}{c}\text { Number of Mapped Dust } \\
\text { Sources }\end{array}$ & Mean Quality Score \\
\hline 1 & 28 & 1.96 \\
\hline 2 & 14 & 2.29 \\
\hline 3 & 17 & 1.94 \\
\hline 4 & 10 & 2.5 \\
\hline 5 & 26 & 2.04 \\
\hline
\end{tabular}


Figure 7. MILLER image (top) depicting a dust event on 16 September 2011 in Southwest Asia and associated dust-plume-head markers mapped by five analysts. The region in the white rectangle is enlarged in the bottom panels. The 1:750,000 (bottom left) and 1:100,000 (bottom right) scale geomorphic landform maps are overlain with mapped plume-head point sources. The ova/ in each panel outlines five points placed by each analyst for single plume head.
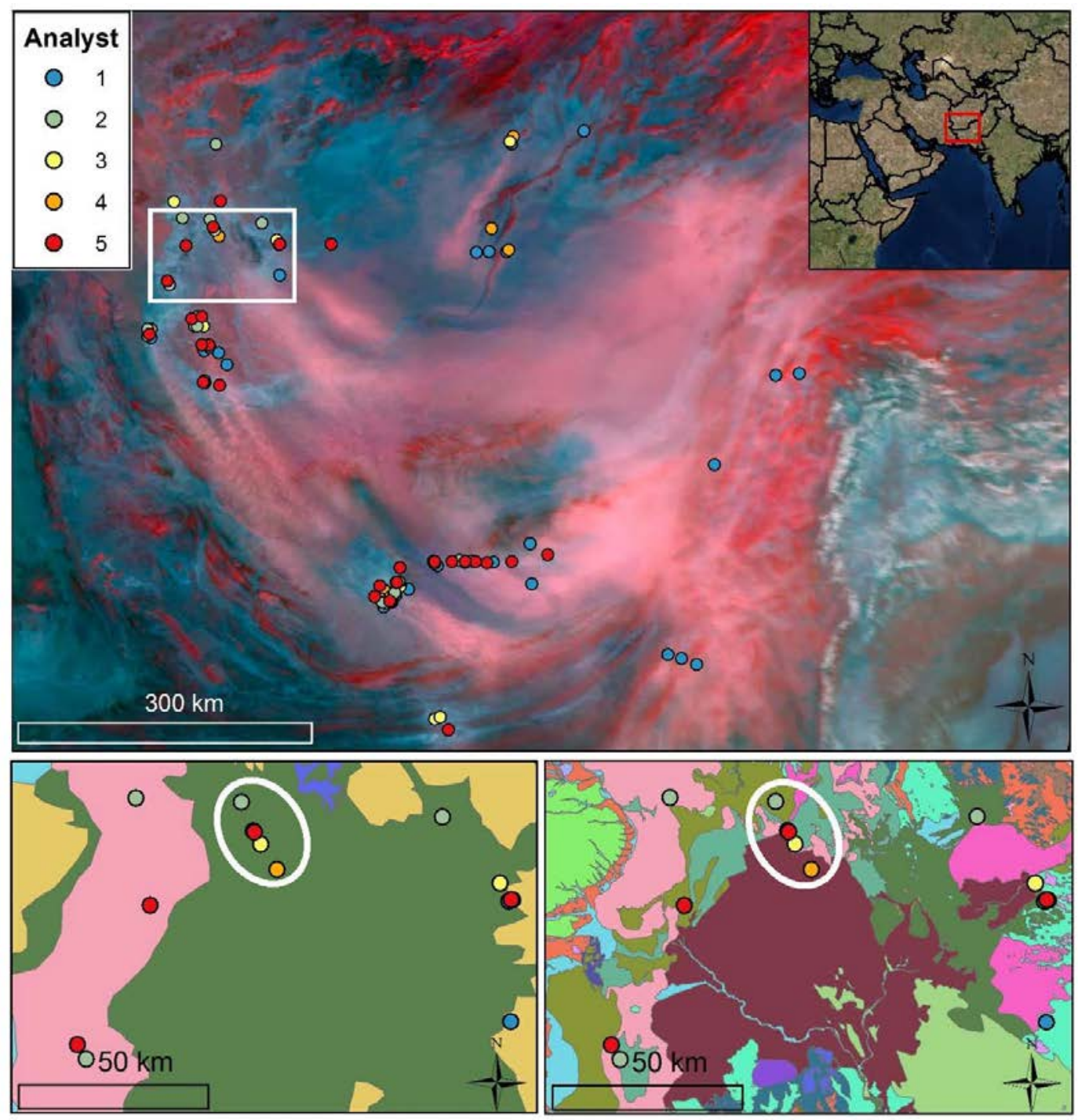

Landform

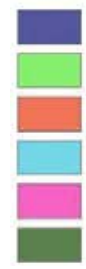
alluvial fan + sand sheet alluvial fan, highly dissected alluvial fan, old alluvial fan, young alluvial plains, old alluvial plains, young

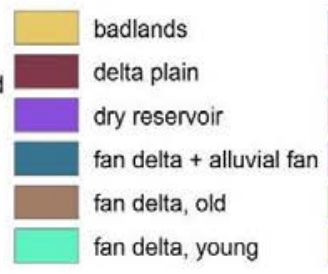

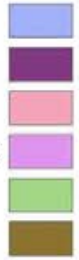

low relief mountains plateau playa (sabkha) salt basin sand seas/dunes sand sheet + fan delta, young sand sheet + plateau sand sheets water wet playa wind erosion features 
Figure 8. Quality scores assigned by each of the five analysts to points mapped in the Southwest Asia case study. Points marked with high confidence (3) are green, moderate confidence (2) are yellow, and low confidence (1) are red. Each panel depicts the points placed by an individual analyst. Note that points mapped by all analysts were assigned a higher quality score (and were therefore easier to identify and map) than those mapped by only one or two analysts.
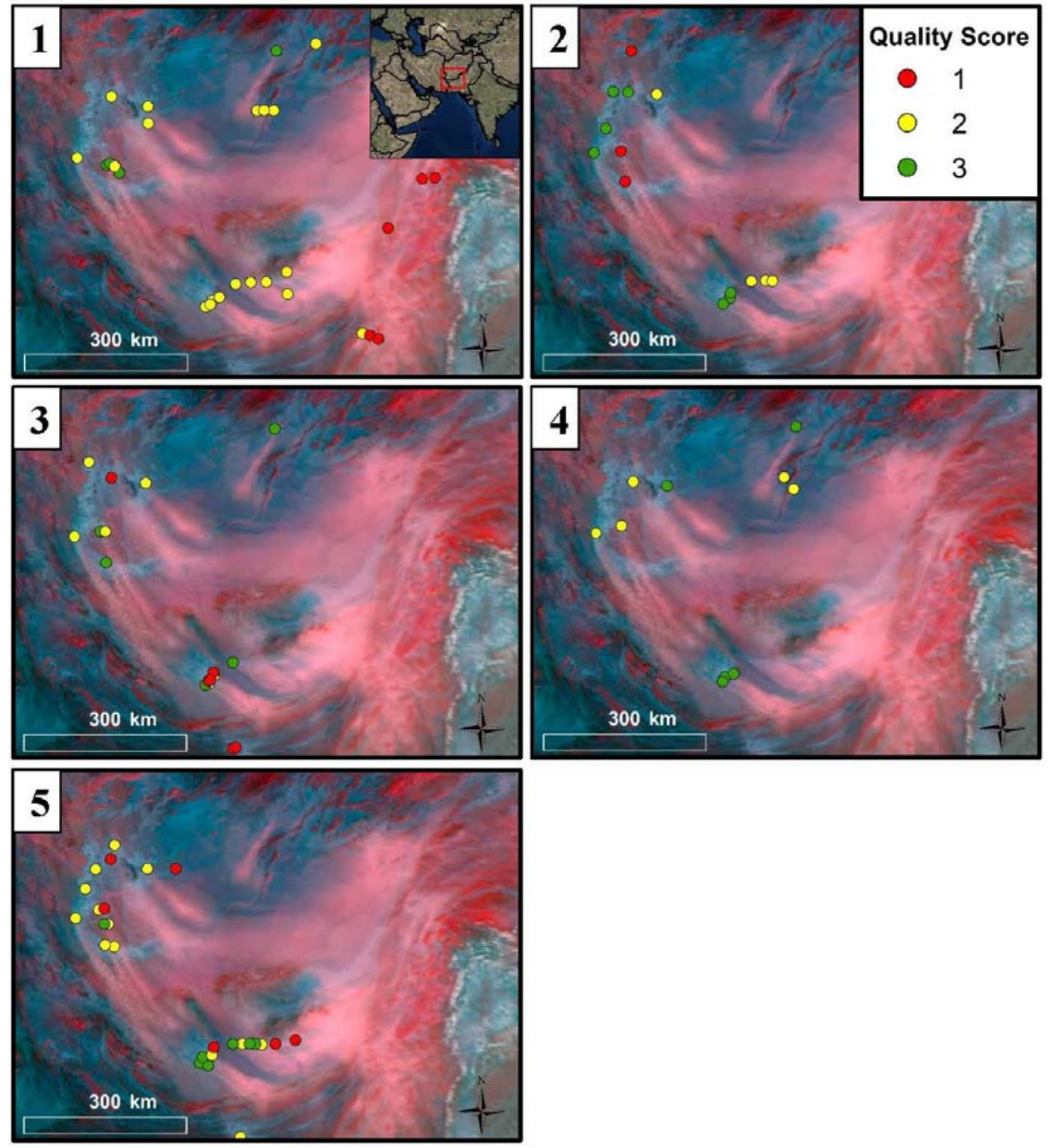


\subsection{Southwest United States}

Table 3, Figure 9, and Figure 10 show results from the second reproducibility experiment conducted for the Southwest United States. One of the team analysts could not participate in the second experiment, so only four of the five initial analysts mapped this region. As shown in Table 3, the number of plume-head point sources mapped by each analyst ranged from 13 to 29 , with an average of $22 \pm 6$.7. Mean quality scores between analysts ranged from 1.23 to 2.14 , with an average of $1.77 \pm 0.41$. In total, there were seven plume heads that all four analysts mapped. Mapped sources for six of these plume heads fell within a $10 \mathrm{~km}$ buffer, while the other fell within a $25 \mathrm{~km}$ buffer. Of the six plume heads mapped within a $10 \mathrm{~km}$ buffer, three were constrained to a $5 \mathrm{~km}$ buffer and one to a $3 \mathrm{~km}$ buffer. Several points were mapped in regions that were not dust producing areas. One analyst mapped several plume-head like features that were actually thin clouds in the region (see Figure 9). Fortunately, that analyst marked all of those points with a quality score of 1 , indicating a low level of confidence (see Figure 10). Landform maps for this region were not accessible at the time of the experiment, and therefore analysts did not conduct overlay analyses.

Table 3. Number of mapped dust sources and mean quality scores for the Southwest United States 2 December 20032050 UTC case study.

\begin{tabular}{|c|c|c|}
\hline Analyst Identification Number & $\begin{array}{c}\text { Number of Mapped Dust } \\
\text { Sources }\end{array}$ & Mean Quality Score \\
\hline 1 & 29 & 1.66 \\
\hline 2 & 13 & 1.23 \\
\hline 3 & 22 & 2.14 \\
\hline 4 & 24 & 2.04 \\
\hline
\end{tabular}


Figure 9. MILLER image (left) depicting a dust event on 2 April 2003 in the Southwest United States and the associated dust-plume head markers mapped by four analysts. Points mapped by each analyst are represented by different colored circles. Note the five points mapped by analyst 1 in the southeast corner of the image that are incorrectly placed on clouds. The area outlined in the black rectangle is enlarged on the right. a close-up of individual plume-head

point sources mapped by each analyst. Also, note the variability in the number of plume heads subjectively identified by each analyst. Buffers placed around plume heads mapped by all analysts show a high degree of duplicability and are constrained to $3 \mathrm{~km}$ (b/ue circle), $5 \mathrm{~km}$ (green circles), and $10 \mathrm{~km}$ (yellow circles) buffers. This image does not include the $25 \mathrm{~km}$ buffer.

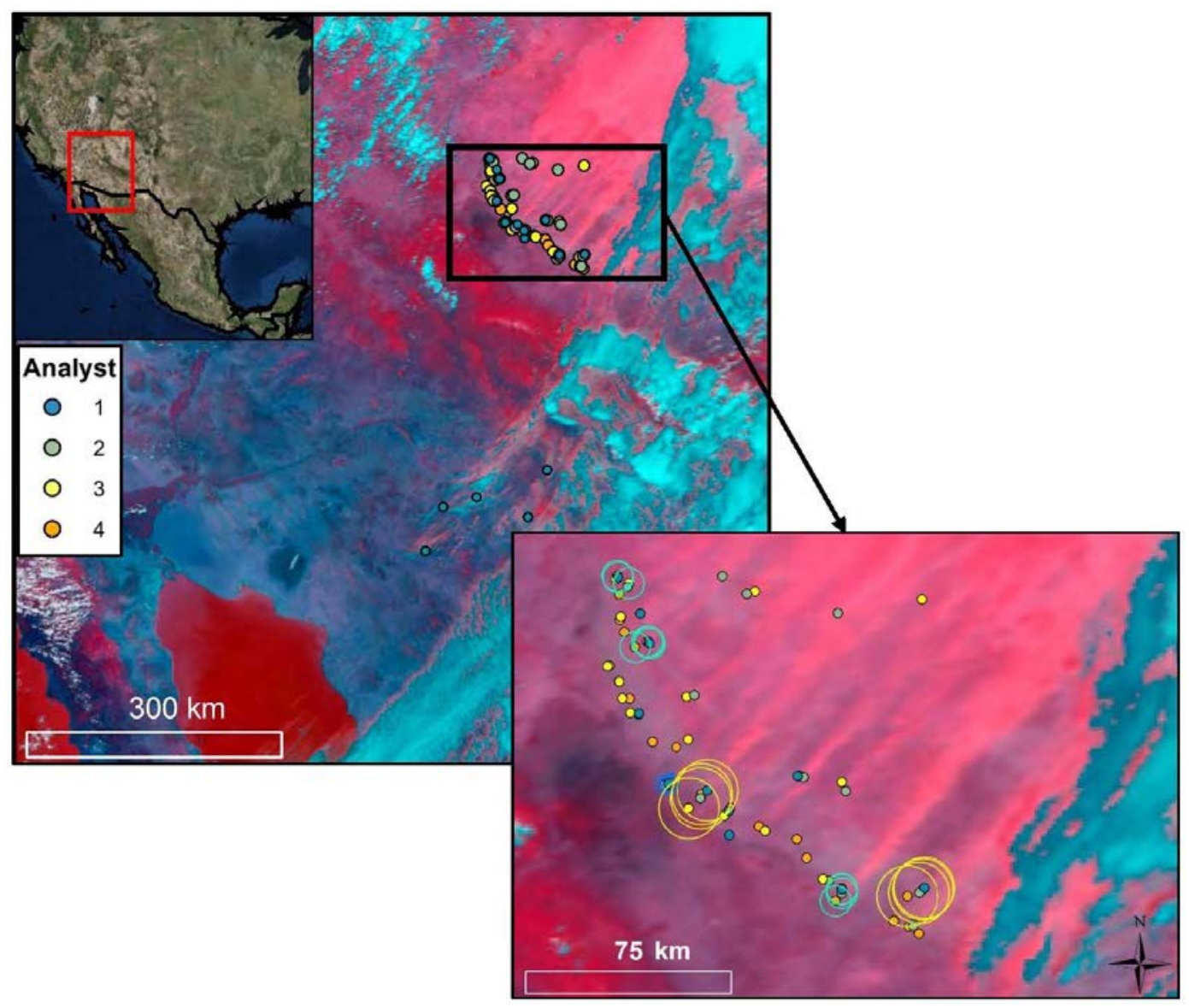


Figure 10. Quality scores assigned by each of the four analysts to points mapped in the Southwest United States case study (shown in Fig. 9). Points marked with high confidence (3) are green, moderate confidence (2) are yellow, and low confidence (1) are red. Each panel depicts the points placed by an individual analyst. As with the Southwest Asia study, plume heads marked by all analysts received a higher quality score than those mapped by just one or two analysts.

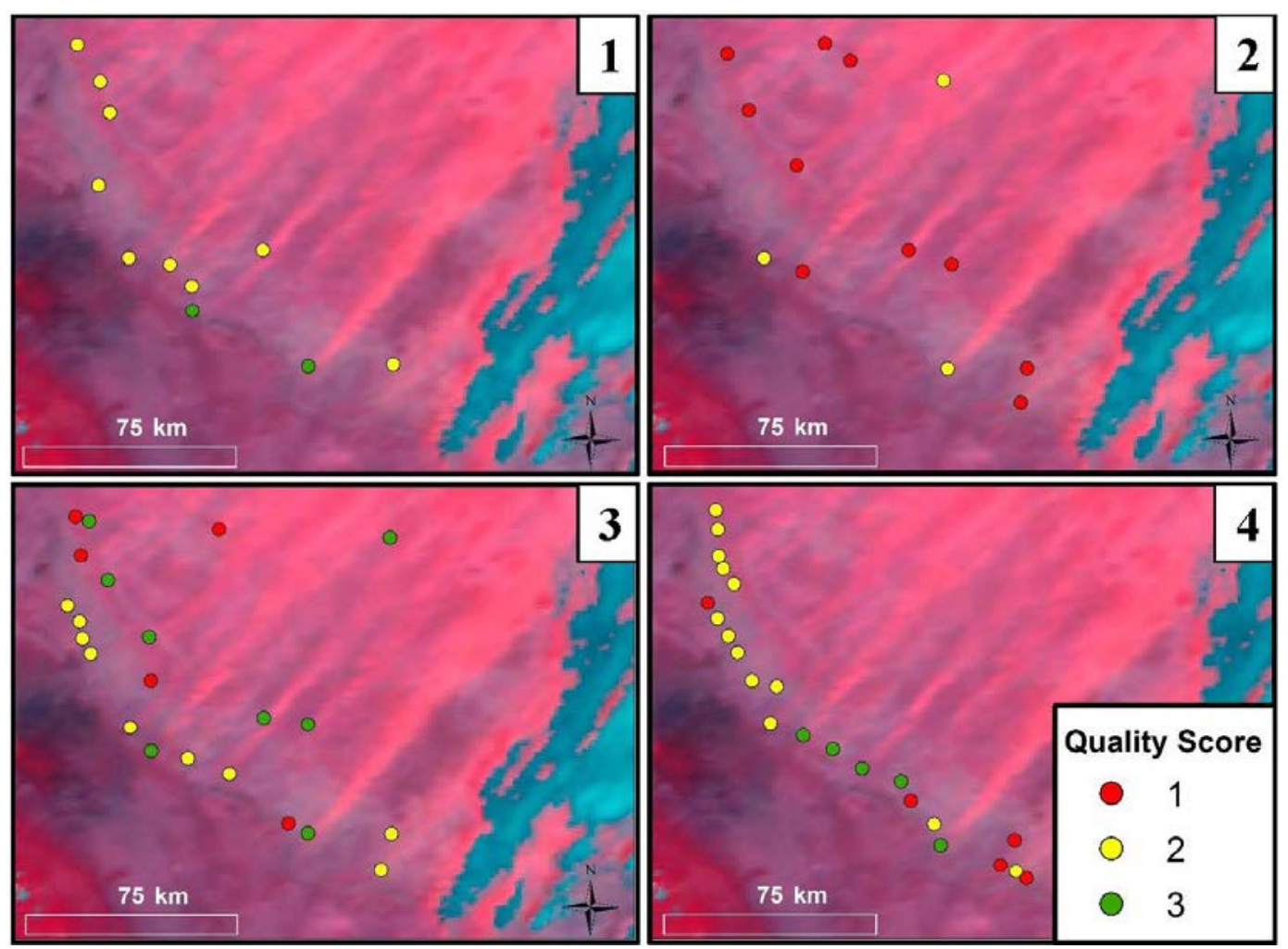




\section{Discussion}

Following the $10 \mathrm{~km}$ location uncertainty assumption per Walker et al. (2009), this reproducibility study considers points to be reproducible if markers submitted by each analyst fall within a $10 \mathrm{~km}$ buffer. In the Southwest Asia case study, only two of the seven plume-head point sources mapped by all participating analysts ( $\sim 29 \%$ ) could be constrained to a $10 \mathrm{~km}$ buffer. From the visual inspection of Figure 7 and from group discussions, however, the team of analysts determined that there are likely more than seven mappable plume-head point sources in the Southwest Asia imagery. In particular, the team agreed in a later group debate that four plume-head point sources that a fraction of the team (three or four analysts) mapped were indeed mappable sources. This result highlights the subjective nature of identifying and mapping plume-head point sources and suggests that analysts do not always define mappable sources in the same manner. It could, therefore, be beneficial to have a team of analysts, rather than a single user, apply this methodology to a given region as point sources overlooked by some team members may be identified and mapped by others.

In the Southwest United States study, six of the seven plume-head point sources mapped by all participating analysts ( $\sim 86 \%)$ could be constrained to a $10 \mathrm{~km}$ buffer. As with the Southwest Asia experiment, group discussions led to an agreement that there are likely more than seven mappable plume-head point sources depicted in the Southwest United States imagery. A thorough group discussion deemed that three plume-head point sources mapped by a fraction of the team (two or three analysts) were mappable sources, though again, there is no way to verify the accuracy of the group consensus.

Our study supports the use of the Walker et al. (2009) methodology for global dust-emission-activity characterization as the analysts were able to successfully map two markedly different, regional-scale desert environments. The results from both the Southwest Asia and U.S. case studies, however, highlight that although the Walker et al. (2009) methodology is reproducible to an extent, it may not produce one-to-one results between multiple analysts. Our findings also demonstrate that dust-source characterization products created via the Walker et al. (2009) methodology may not be suitable proxies for observational "truth." If the definition of reproducible was expanded to include point sources mapped to within a $15 \mathrm{~km}$ 
buffer, however, five of the seven ( $71 \%$ ) Southwest Asia points and all seven (100\%) of the Southwest United States points would be considered reproducible. Thus, aspects of the final product's format and intended application largely affect conclusions about the reproducibility of the Walker et al. (2009) technique. For example, Walker et al. (2009) applied their final dataset to a dust transport model as a preferential dust-source region map interpolated to a $27 \mathrm{~km}$ grid. This grid spacing resolution is well outside the bounds of expected detection and analyst-induced location-error uncertainty.

Of the 183 total points mapped by the analysts in this study, only five were deemed to be incorrectly placed at definitively non-dust-emission sources (see Figure 9). This demonstrates that users encountered minimal difficulty in differentiating between plume-head point-source locations and false-positive "plume-head lookalikes," like forest fires or high-relief terrain. The five points that were incorrectly mapped as plume-head point sources (and were in fact clouds) were assigned a quality score of 1 , which indicates that the quality score is an important aspect of this methodology that can be used to winnow out unusable data. Plume-head point sources mapped by one or two analysts typically received a quality score of 1 , while 89\% (in Southwest Asia) and 79\% (in the Southwest United States) of plume-head point sources mapped by all analysts received a quality score of 2 or 3 (see Figures 8 and 10). User confidence could therefore be used as a predictive measure to identify mapped points that are likely actual dustemission sources.

Our geomorphic overlay assessment suggests that the $1 \mathrm{~km}$ resolution of MODIS imagery coupled with the mapped point-source location uncertainty may prohibit use of the Walker et al. (2009) technique for linking dust-emission sources to a particular landform type or geographic location outside of very coarse-resolution scale analyses. Previous studies have shown that individual geomorphic landforms may not always act as homogeneous dust-emission sources and may consist of both erodible and unerodible portions (Walker et al. 2009; King et al. 2011; Sweeney et al. 2011). Additionally, some dust-emission sources may be associated with individual fields or farmland (e.g., Gillette 1999). Dust plumes emitted from these relatively small, localized sources are likely too narrow for MODIS sensors to detect. 


\section{Opportunities for Future Research}

The inability of the MODIS data to display narrow dust plumes is a limiting factor when characterizing dust-emission sources for highly variable landscapes. Newer and higher-resolution multispectral satellite capabilities, such as the Visible Infrared Imager Radiometer Suite (VIIRS), may be able to ameliorate this issue. Future studies aiming to map dust-emission sources using an approach similar to the Walker et al. (2009) methodology should consider use of these higher-resolution satellite platforms to enhance dust-emission potential associations to various landscape attributes. Additionally, MODIS is currently operating past its initial design life of six years, so continued dependency on image acquisition from MODIS could pose a risk. The significant drawback to any new satellite capability, however, is a substantial reduction in the length of the record. VIIRS, for example, was launched in 2011 while MODIS was launched in 2000 and can therefore provide a longer record of continuous data.

A second limitation of the approach used in this study is the fact that MODIS visible-channel imagery can only be acquired during daylight hours. Satellite orbit timing could also affect dust-level estimations as diurnal patterns of blowing dust exist due to the heterogeneous heating of the atmosphere and land surface (Stout 2010). If a satellite does not pass over the area of interest during times with the greatest frequency of dust storms, it could miss the dust events (particularly if short lived) entirely. For example, Orgill and Sehmel (1976) examined dust storms reported in weather station data in the United States and determined that the majority of dust storms in the region occur between 1200 and 2000. This time period only partially aligns with MODIS satellite pass over in the Southwest United States, which is always between 1700 and 2100 UTC.

The team of CRREL analysts will conduct future research efforts using the Walker et al. (2009) methodology to better understand how temporal and spatial patterns of dust emissivity relate to local climate patterns and regional geomorphology. The analysts who participated in this study will build a dust-plume-head point source database using the Walker et al. (2009) technique and 16 continuous years of MODIS data for U.S. and Mexico desert regions. Findings from this future study will help guide data interpretation and collaborative mapping approaches. 


\section{References}

Adams, D. K., and A. C. Comrie. 1997. The North American Monsoon. Bulletin of the American Meteorological Society 78 (10): 2197- 2213.

Al-Hemoud, A., M. Al-Sudairawi, S. Neelamanai, A. Naseeb, and W. Behbehani. 2017. Socioeconomic Effect of Dust Storms in Kuwait. Arabian J ournal of Geosciences 10 (1): 1-9.

Affleck, R., P. Seman, M. Deegan, R. Freeman, and S. Sargand. 2011. Documenting Lessons Learned in Afghanistan Concerning Design and Construction Challenges. ERDC/ CRREL TR-11-02. Hanover, NH: U.S. Army Engineer Research and Development Center.

Bacon, S. N., and E. V. McDonald. 2016. Regional Distribution of Salt-Rich Dust Across Southwest Asia Based on Predictive Soil-Geomorphic Mapping Techniques. In Military Geosciences and Desert Warfare, ed. E. V. McDonald and T. Bullard, 237- 56. New York, NY: Springer.

Baddock, M. C., J. E. Bullard, and R. G. Bryant. 2009. Dust Source Identification Using MODIS: A Comparison of Techniques Applied to the Lake Eyre Basin, Australia. Remote Sensing of Environment 113 (7): 1511- 28.

Baddock, M. C., P. Ginoux, J . E. Bullard, and T. E. Gill. 2016. Do MODIS-Defined Dust Sources Have a Geomorphological Signature? Geophysical Research Letters 43 (6): 2606- 2613.

Breckle, S.-W. 2007. Flora and Vegetation of Afghanistan. Basic and Applied Dryland Research 1(2): 155-94.

Brindley, H., P. Knippertz, C. Ryder, and I. Ashpole. 2012. A Critical Evaluation of the Ability of the Spinning Enhanced Visible and Infrared Imager (SEVIRI) Thermal Infrared Red-Green-Blue Rendering to Identify Dust Events: Theoretical Analysis. Journal of Geophysical Research: Atmospheres 117 (D7): D07201. doi:10.1029/2011J D017326.

Bullard, J . E., M. C. Baddock, G. McTainsh, and J . Leys. 2008. Sub-Basin Scale Dust Source Geomorphology Detected Using MODIS. Geophysical Research Letters 35 (15): L15404. doi:10.1029/2008GL033928.

Bullard, J . E., S. P. Harrison, M. C. Baddock, N. Drake, T. E. Gill, G. McTainsh, and Y. Sun. 2011. Preferential Dust Sources: A Geomorphological Classification Designed for Use in Global Dust-Cycle Models. J ournal of Geophysical Research 116 (F4): F04034. doi:10.1029/2011J F002061.

De Longueville, F., Y.-C. Hountondji, S. Henry, and P. Ozer. 2010. What Do We Know About Effects of Desert Dust on Air Quality and Human Health in West Africa Compared to Other Regions? Science of the Total Environment 409 (1): 1-8.

EPA (U.S. Environmental Protection Agency). 2012. Ecoregions of North America. http://maps4.arcgisonline.com/ArcGIS/rest/services/DOI/EPA_Ecoregions_of_North_America LMapServer (accessed 23 September 2016).

Gillette, D. A. 1999. A Qualitative Geophysical Explanation for Hot Spot Dust Emitting Source Regions. Contributions to Atmospheric Physics 72 (1): 67- 77. 
Ginoux, P., J. M. Prospero, T. E. Gill, N. C. Hsu, and M. Zhao. 2012. Global-Scale Attribution of Anthropogenic and Natural Dust Sources and Their Emission Rates Based on MODIS Deep Blue Aerosol Products. Reviews of Geophysics 50 (3): RG3005. doi:10.1029/2012RG000388.

Goudie, A., and N. J . Middleton. 2006. Desert Dust in the Global System. New York: Springer.

Huang, J., T. Wang, W. Wang, Z. Li, and H. Yan. 2014. Climate Effects of Dust Aerosols over East Asian Arid and Semiarid Regions. J ournal of Geophysical Research: Atmospheres 119 (19): 11,398-11,416.

Jafari, R., and M. Malekian. 2015. Comparison and Evaluation of Dust Detection Algorithms Using MODIS Aqua/ Terra Level 1B Data and MODIS/OMI Dust Products in the Middle East. International J ournal of Remote Sensing 36 (2): 597-617.

Khaurin, H. H. 2003. Trees and Bushes of Afghanistan. Kabul, Afghanistan: Food and Agriculture Organization, Forest National Coordination Office. http://eafghanag.ucdavis.edu/c_livestock/Man_For_Fodder_Trees_Afghan_FA0.pdf (accessed 01 February 2017).

King, J ., V. Etyemezian, M. Sweeney, B. J . Buck, and G. Nikolich. 2011. Dust Emission Variability at the Salton Sea, California, USA. Aeolian Research 3 (1): 67- 79.

Knippertz, P., and J .-B. W. Stuut, ed. 2014. Mineral Dust: A Key Player in the Earth System. New York: Springer.

Lary, D. J ., A. H. Alavi, A. H. Gandomi, and A. L. Walker. 2016. Machine Learning in Geosciences and Remote Sensing. Geoscience Frontiers 7 (1): 3- 10.

Legrand, M., C. N'doume, and I. J ankowiak. 1994. Satellite-Derived Climatology of the Saharan Aerosol. In Passive Infrared Remote Sensing of Clouds and the Atmosphere II, ed. D. K. Lynch, 127- 35. Proceedings of SPIE International Society of Optical Engineering.

Lensky, I. M., and D. Rosenfeld. 2008. Clouds-Aerosols-Precipitation Satellite Analysis Tool (CAPSAT). Atmospheric Chemistry and Physics 8 (22): 6739- 53.

Liu, M., D. L. Westphal, A. L. Walker, T. R. Holt, K. A. Richardson, and S. D. Miller. 2007. COAMPS real-Time Dust Storm Forecasting During Operation Iraqi Freedom. Weather and Forecasting 22 (1): 192- 206.

Mahowald, N. M., A. R. Baker, G. Bergametti, N. Brooks, R. A. Duce, T. D. Jickells, N. Kubilay, J . M. Prospero, and I. Tegen. 2005. Atmospheric Global Dust Cycle and Iron Inputs to the Ocean. Global Biogeochemical Cycles 19 (4): GB4025. doi:10.1029/2004GB002402.

Mahowald, N., S. Albani, J . F. Kok, S. Engelstaeder, R. Scanza, D. S. Ward, and M. G. Flanner. 2014. The Size Distribution of Desert Dust Aerosols and Its Impact on the Earth System. Aeolian Research 15:53- 71.

McDonald, E., S. Bacon, S. Baker, R. Amit, J . L. Antinao, M. Berli, T. Bullard, T. Caldwell, O. Crouvi, and Y. Enzel. 2013. Integrated Desert Terrain Forecasting for Military Operations. Reno, NV: Desert Research Institute. 
Middleton, N. J . 2017. Desert Dust Hazards: A Global Review. Aeolian Research 24:5363.

Miller, S. D. 2003. A Consolidated Technique for Enhancing Desert Dust Storms with MODIS. Geophysical Research Letters 30 (20): 2071. doi:10.1029/2003GL018279.

Moridnejad, A., N. Karimi, and P. A. Ariya. 2015. A New Inventory for Middle East Dust Source Points. Environmental Monitoring and Assessment 187 (9): 1- 11.

Muhs, D. R., J. M. Prospero, M. C. Baddock, and T. E. Gill. 2014. Identifying Sources of Aeolian Mineral Dust: Present and Past. In Mineral Dust, ed. P. Knippertz and J .B. W. Stuut, 51- 74. New York: Springer.

Nadolski, V. L. 1998. Automated Surface Observing System (ASOS) User's Guide. Silver Spring, MD: National Oceanic and Atmospheric Administration. http://www.nws.noaa.gov/asos/pdfs/aum-toc.pdf (accessed 11 November 2016).

Okin, G. S., J . E. Bullard, R. L. Reynolds, J .-A. C. Ballantine, K. Schepanski, M. C. Todd, J. Belnap, M. C. Baddock, T. E. Gill, and M. E. Miller. 2011. Dust: Small-Scale Processes with Global Consequences. EOS, Transactions American Geophysical Union 92 (29): 241.

Orgill, M. M., and G. A. Sehmel. 1976. Frequency and Diurnal Variation of Dust Storms in the Contiguous USA. Atmospheric Environment 10 (10): 813- 25.

Prospero, J . M., P. Ginoux, O. Torres, S. E. Nicholson, and T. E. Gill. 2002. Environmental Characterization of Global Sources of Atmospheric Soil Dust Identified with the NIMBUS 7 Total Ozone Mapping Spectrometer (TOMS) Absorbing Aerosol Product. Reviews of Geophysics 40 (1): 2-1-2-31.

Rashki, A., D. G. Kaskaoutis, A. S. Goudie, and R. A. Kahn. 2013. Dryness of Ephemeral Lakes and Consequences for Dust Activity: The Case of the Hamoun Drainage Basin, Southeastern Iran. Science of the Total Environment 463 (1): 552- 564.

Ravi, S., P. D’Odorico, D. D. Breshears, J. P. Field, A. S. Goudie, T. E. Huxman, J . Li, G. S. Okin, R. J . Swap, A. D. Thomas, S. Van Pelt, J . J . Whicker, and T. M. Zobeck. 2011. Aeolian Processes and the Biosphere. Reviews of Geophysics 49 (3): RG3001. doi:10.1029/2010RG000328.

Rushing, J . F., and J . S. Tingle. 2006. Dust Control Field Handbook: Standard Practices for Mitigating Dust on Helipads, Lines of Communication, Airfields, and Base Camps. ERDC/ GSL SR-06-7. Vicksburg, MS: U.S. Army Engineer Research and Development Center.

Rushing, J . F., A. Harrison, and J . S. Tingle. 2005. Evaluation of Application Methods and Products for Mitigating Dust for Lines-of-Communication and Base Camp Operations. ERDC/ GSL TR-05-9. Vicksburg, MS: U.S. Army Engineer Research and Development Center.

Shepherd, G., E. Terradellas, A. Baklanov, U. Kang, W. Sprigg, S. Nickovic, A. D. Boloorani, A. Al-Dousari, S. Basart, and A. Benedetti. 2016. Global Assessment of Sand and Dust Storms. Nairobi: United Nations Environment Programme. 
Shinn, E. A., G. W. Smith, J . M. Prospero, P. Betzer, M. L. Hayes, V. Garrison, and R. T. Barber. 2000. African Dust and the Demise of Caribbean Coral Reefs. Geophysical Research Letters 27 (19): 3029- 32.

Skiles, S. M., T. H. Painter, J . Belnap, L. Holland, R. L. Reynolds, H. L. Goldstein, and J. Lin. 2015. Regional Variability in Dust-on-Snow Processes and Impacts in the Upper Colorado River Basin. Hydrological Processes 29 (26): 5397- 5413.

Sprigg, W. A., S. Nickovic, J . N. Galgiani, G. Pejanovic, S. Petkovic, M. Vujadinovic, A. Vukovic, M. Dacic, S. DiBiase, A. Prasad, and H. El-Askary. 2014. Regional Dust Storm Modeling for Health Services: The Case of Valley Fever. Aeolian Research 14:53- 73.

Stout, J . E. 2010. Diurnal Patterns of Blowing Sand. Earth Surface Processes and Landforms 35 (3): 314- 18.

Sweeney, M. R., and J oseph A. Mason. 2013. Mechanisms of Dust Emission from Pleistocene Loess Deposits, Nebraska, USA. J ournal of Geophysical Research: Earth Surface 118 (3): 1460- 71.

Sweeney, M. R., E. V. McDonald, and V. Etyemezian. 2011. Quantifying Dust Emissions from Desert Landforms, Eastern Mojave Desert, USA. Geomorphology 135 (1- 2): 21-34.

Sweeney, M. R., H. Lu, M. Cui, J . A. Mason, H. Feng, and Z. Xu. 2016. Sand Dunes as Potential Sources of Dust in Northern China. Science China Earth Sciences 59 (4): $760-69$.

Tong, D. Q., M. Dan, T. Wang, and P. Lee. 2012. Long-Term Dust Climatology in the Western United States Reconstructed from Routine Aerosol Ground Monitoring. Atmospheric Chemistry and Physics 12 (11): 5189- 5205.

Torres, O., P. K. Bhartia, J . R. Herman, Z. Ahmad, and J . Gleason. 1998. Derivation of Aerosol Properties from Satellite Measurements of Backscattered Ultraviolet Radiation: Theoretical Basis. J ournal of Geophysical Research: Atmospheres 103 (D14): 17099- 110.

Walker, A. L., M. Liu, S. D. Miller, K. A. Richardson, and D. L. Westphal. 2009. Development of a Dust Source Database for Mesoscale Forecasting in Southwest Asia. J ournal of Geophysical Research 114 (D18): D18207. doi:10.1029/2008J D011541.

Wang, F., X. Zhao, C. Gerlein-Safdi, Y. Mu, D. Wang, and Q. Lu. 2017. Global Sources, Emissions, Transport and Deposition of Dust and Sand and Their Effects on the Climate and Environment: A Review. Frontiers of Environmental Science and Engineering 11(1): 1- 9. doi:10.1007/ s11783-017-0904-z.

Webb, N. P., A. Chappell, C. L. Strong, S. K. Marx, and G. H. McTainsh. 2012. The Significance of Carbon-Enriched Dust for Global Carbon Accounting. Global Change Biology 18 (11): 3275- 78.

Zhang, X., D. Q. Tong, G. Wu, X. Wang, A. Xiu, Y. Han, T. Xu, S. Zhang, and H. Zhao. Forthcoming. Identification of Dust Sources and Hotspots in East Asia During 2000-2015: Implications for Numerical Modeling and Forecasting. Atmospheric Chemistry and Physics Discussions. doi:10.5194/ acp-2016-681. 


\section{Appendix A: Obtaining ASOS Data}

To obtain weather station data for a particular area of interest, go to https://mesonet.agron.iastate.edu/request/download.phtml?network=AZ_ASOS. Look for "Select Network" at the top of the page, choose a region of interest, and click "Switch to Network." Section 1, "Select Station/ Network by Clicking on Location," shows a map of the selected area with red markers denoting the location of each ASOS station in the region. Above the map, each station is listed alphabetically under "Sort Available Stations." From this list, choose several stations that cover the area of interest and click "Add Selected." It is not necessary to download data from every available station, particularly in regions where an abundance exist; but stations both within and immediately outside of the area of concern should be downloaded to obtain comprehensive coverage of the region. The chosen stations will appear in the "Selected Stations" box. In section 2, "Select from Available Data," select "All Available" to include all measurements and observations from the ASOS stations. In section 3, "Specific Date Range," select the desired range of interest. In section 4, "Time Zone of Observation Times," select UTC to avoid potential problems with regions that span across multiple time zones. In section 5, "Download Options," select tab delimited, include the latitude and longitude data, and change "View result data in web browser" to "Save result data to file on computer." After making these selections, click "Get Data."

Once downloaded, the data will need to undergo some minor processing to alter the date and time stamps associated with each entry and to remove unwanted fields. Software engineers at Atmospheric and Environmental Research, Inc., developed a Python script to handle these processing steps. Use the Python script entitled "ASOS_Processing.py" (included below), and type the following command: python ASOS_Processing.py /path/to/directory, where /path/to/directory is replaced with the correct path name. Each processed weather station dataset produces a text (.txt) and a comma-separated values (.csv) file. 


\section{ASOS Processing Python Script:}

' ' '

- - -

Title: ASOS_Processing.py

Date Created: December 2015

Updated: March 2017

Outputs: For each weather station, a text file with all hourly reports processed --> <inputfile>_PROCESSED.txt

For all weather stations processed in a single batch, a summary.xls file with only reports from "events" (defined below) included --> ASOS_STATS_events_only.xls

$--$

\# PURPOSE

\#- - - - - - -

ASOS_Processing.py processes weather station data files downloaded from the Iowa Environmental Mesonet ASOS Network. The URL for the IEM ASOS archive is: https://mesonet.agron. iastate.edu/request/download.phtml?network=AZ_ASOS

This script can process a single file or a batch of data files contained in the same directory. This script will produce 1) a text file of processed data for each raw data file it is given and 2) a summary.xls file that compiles all reports associated with decreased visibility or obscurations reported.

\# SUMMARY

ASOS_processing.py reads data files downloaded from the IEM ASOS Network and processes them by scanning for missing values, changing the data type of the time stamp field, removing unwanted fields, and searching for the occurrence of visibility obscurations and thunderstorms (which are logged in a new field). The processed data is saved in a new tab delimited text file with the input file name appended with the suffix _PROCESSED.txt.

For each weather station data file:

input file = my_data.txt

output file = my_data_PROCESSED.txt

Events summary file compilation:

Events are defined as reports that contain decreased visibility conditions ( $<7$ nautical miles), reports of obscuration (blowing dust (BLDU), dust storm (DS), sand storm (SS), dust whorls or dust devils (PO), haze (HZ), fog/mist, or smoke), or a combination of the two.

event_code Obscuration and Visibility Condition

$0.0=$ no obscurations and high visibility

1.0 = low visibility, no obscuration reported

$2.0=\quad$ low visibility and BLDU, DS, SS, PO

$2.1=$ high visibility and BLDU, DS, SS, PO

$3.0=$ low visibility and haze

$3.1=$ high visibility and haze

$8.0=\quad$ low visibility and fog $/ \mathrm{mist}$

$8.1=$ high visibility and $\mathrm{fog} / \mathrm{mist}$

$9.0=\quad$ low visibility and smoke

$9.1=$ high visibility and smoke

All reports are assigned an event_code. All reports with an event code that is not equal to $\odot . \odot$ are compiled to remove duplicate reports of the same event on the same day. Therefore, 
for a given station, only one report of an event is recorded per day (this is for calculating statistics of the occurrence of events).

This compilation is saved in: ASOS_STATS_events_only.xls

\# USAGE

\#- - - - - - -

To run this processing script, place a copy of this code in a directory with the data file(s) that need to be processed. In Terminal, navigate to the directory that contains the script and data file(s), then enter this command:

python ASOS_Processing.py /path/to/directory/with/data/files

Where you will replace the /path/to/directory... with the correct path name. The script will print updates and information to the terminal window about the processing. This script will create, use, and then delete a temporary file in the directory that holds the script and your data file to be processed. The processed output files will be saved to this same working directory.

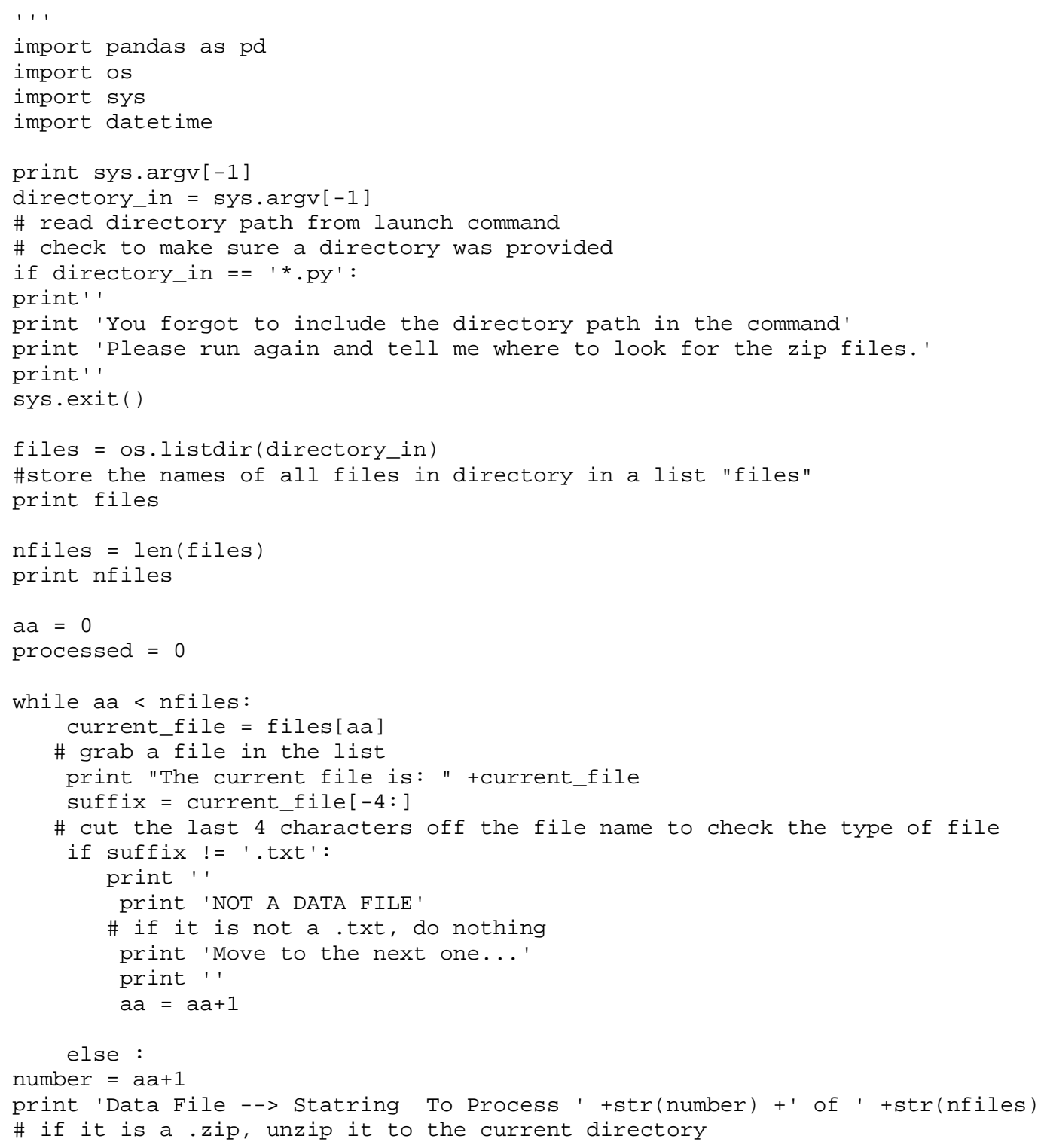


raw_input_file = directory_in+'/'+current_file

output_file = 'ASOS_STATS_events_only.xls'

\# generate output file name

\# Need to strip the first 5 lines of the input file downloaded from IEM ASos archive numline $=5$

\# 5 lines to skip

$\mathrm{p}="$ "

o=open("temp_output.txt", "a")

$f=o p e n$ (raw_input_file)

for $i$ in range(numline):

$f \cdot \operatorname{next}()$

for line in $f$ :

if $p$ :

$0 . \operatorname{write}(p)$

$\mathrm{p}=$ line

f.close ()

$0 . \operatorname{close}()$

\# open data file with first 5 lines removed to read the data, skip spaces after tab delimiters

df_in = pd.read_csv('temp_output.txt', delimiter='\t', skipinitialspace=True, )

\# read data from file into pandas Data Frame

os.remove('temp_output.txt')

\# delete the intermediate file with the top 5 lines stripped off

print 'Beginning Data Processing'

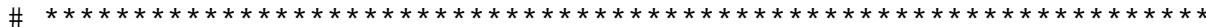

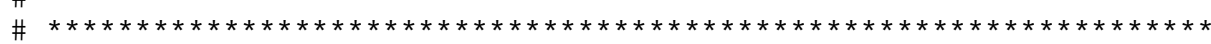

\# DATA PROCESSING - carried out in order of the parameter columns from input data file \# Station Name --> Add "K" to station text string to match shapefile names.

add_str $=$ ' $\mathrm{K}$ '

for $i$ in range $\left(\odot\right.$, len $\left.\left(d f \_i n . i n d e x\right)\right)$ :

existing_str $=d f \_i n . l o c[i$, 'station']

df_in.set_value(i, 'station', add_str+existing_str)

\# Time Stamp Processing --> Convert 'valid' from string to type = datetime

df_in['valid'] = pd.to_datetime(df_in['valid'])

\# For Numeric Data -- Scan for missing values (marked with "M" by IEM ASOS data

interpreter) and assign a value of "null" (without "") if found

\# Also add hooks for unit conversion

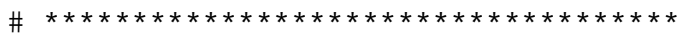

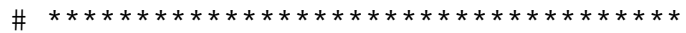

\# Define a function to look at columns that should have numeric values, but they are mixed in with strings ("M") from the IEM ASOS interpreter for missing values

def is_number(s):

try:

float (s)

return True

except ValueError:

return False

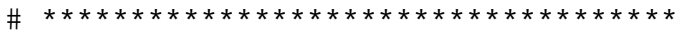

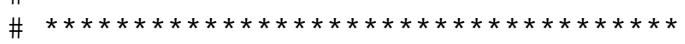

\# Check the Data, Replace M's from IEM ASOS interp. with '' (no data) so that ArcGIS reads the column in as a float and the empty cells will become <Null> values

\#

df_in ['hour'] = pd.Series $\left(\odot\right.$, index $\left.=d f \_i n . i n d e x\right)$

\# create the new column

df_in ['day'] = pd.Series $(\odot$, index=df_in.index $)$ 


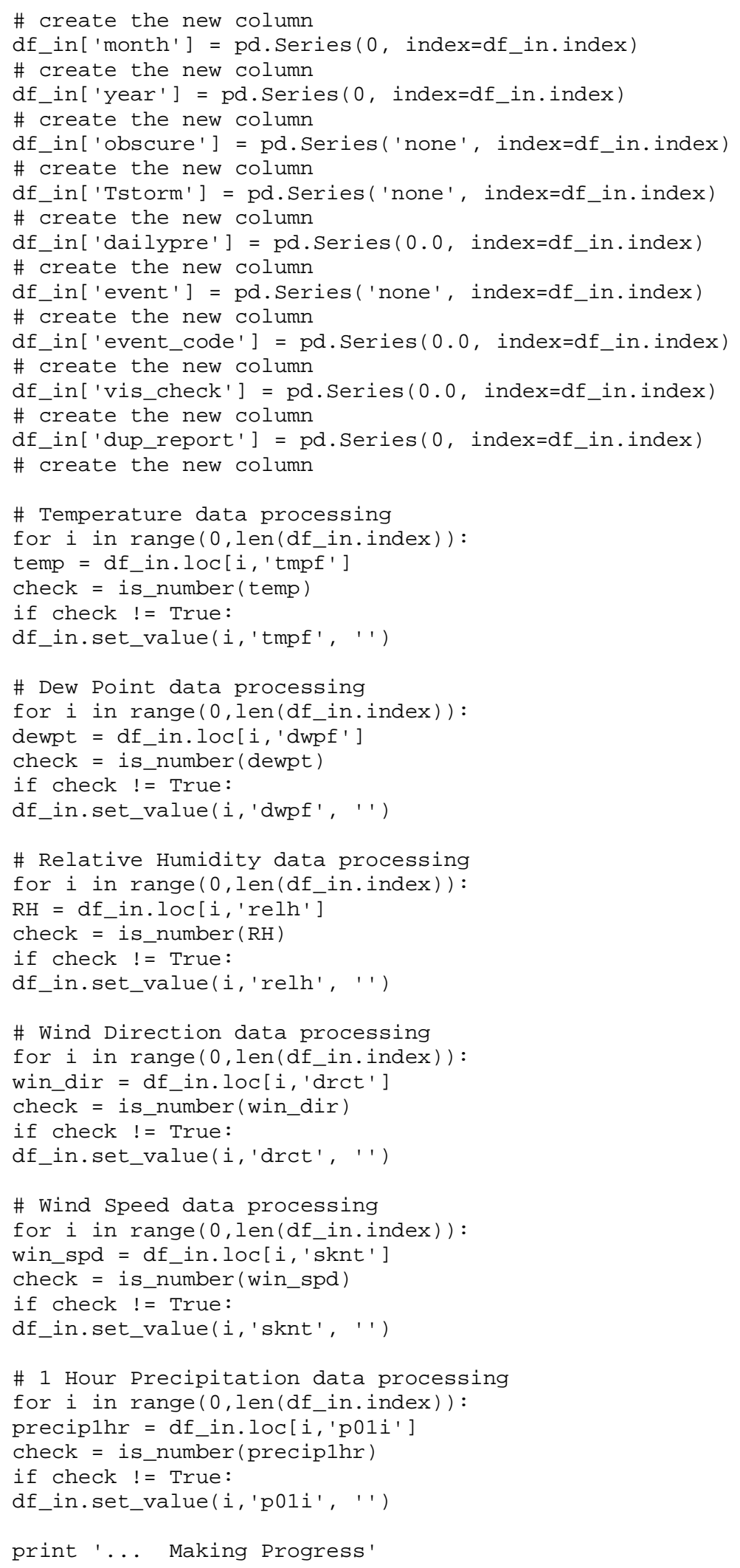


for $i$ in range $\left(\odot\right.$, len $\left.\left(d f \_i n . i n d e x\right)\right)$ :

test_val $=d f \_i n .10 c[i, ' a l t i ']$

check = is_number(test_val)

if check != True:

df_in.set_value(i, 'alti', ' ')

\# Sea Level Pressure data processing

for $i$ in $r$ ange $(\odot$, len $(d f$ in.index $))$ :

test_val $=\mathrm{df}$ in.loc[i, 'mslp']

check = is_number (test_val)

if check != True:

df_in.set_value(i, 'mslp', ' ')

\# Visibility data processing

for $i$ in range $\left(\odot\right.$, len $\left.\left(d f \_i n . i n d e x\right)\right)$ :

vis $=d f$ in.loc $[i$, 'vsby']

check = is_number (vis)

if check != True:

df_in.set_value(i, 'vis_check', 99.0)

else:

df_in.set_value(i, 'vis_check', vis)

\# Gust Wind speed data processing

for $i$ in range $(\odot$, len $(d f$ in.index $))$ :

gust_val $=$ df_in.loc $[i$, 'gust']

\#print gust_val

check = is_number (gust_val)

if check != True:

df_in.set_value(i, 'gust', ' ')

print '... ... Almost Done'

print ' '

\# search METAR string for different obscuration codes and store them in a new column called "obscure"

for $i$ in range $(\odot$, len (df_in.index)):

input $=d f$ in.loc $[i$, 'valid']

year $=$ input $\cdot$ year

month = input.month

day = input.day

hour = input .hour

df_in.set_value( $i$, 'year', year)

df_in.set_value(i, 'month', month)

df_in.set_value( $i$, 'day', day)

df_in.set_value( $i$, 'hour', hour)

\# Loop through the METAR strings, ask if any of the obscuration or storm codes are in each \# Individual METAR string, if so, change the value in the "obscure" or "Tstorm" fields

for $i$ in range $\left(\odot\right.$, len $\left.\left(d f \_i n . i n d e x\right)\right)$ :

metar $=d f \_i n . \operatorname{loc}\left[i,{ }^{\prime}\right.$ metar']

\# Get row i of METAR

BLDU = metar.find ('BLDU' )

\# Search for "BLDU" and log the location

DSNT = metar.find ('DSNT')

$\mathrm{DS}=$ metar.find('DS')

ALQDS = metar.find ('ALQDS')

$\mathrm{RMK}=$ metar.find $($ 'RMK')

$\mathrm{PO}=$ metar.find ('PO')

$\mathrm{HZ}=$ metar.find $\left(' H Z{ }^{\prime}\right)$

fog $=$ metar.find ('FG')

smoke = metar.find ('FU' $)$

$\mathrm{SA}=\operatorname{metar}$. find $\left({ }^{S} \mathrm{~A}^{\prime}\right)$

mist $=$ metar.find $($ 'BR' $)$

tstorm $=$ metar.find ('TS')

zap $=$ metar.find $($ 'LTG') 
fropa $=$ metar.find ('FROPA')

$\mathrm{dp}=\operatorname{metar}$. find $\left({ }^{\prime} 70^{\prime}\right)$

if BLDU $!=-1$ :

df_in.set_value( $i$, 'obscure', 'Blowing Dust')

df_in.set_value( $i$, 'event', 'dust/sand obs')

\# Continue adding the text strings

elif PO != -1:

df_in.set_value(i, 'obscure', 'Dust Devils')

df_in.set_value( $i$, 'event', 'dust/sand obs')

elif $\mathrm{HZ} !=-1$ :

df_in.set_value(i, 'obscure', 'Haze')

df_in.set_value( $i$, 'event', 'haze')

elif fog $!=-1$ :

df_in.set_value(i, 'obscure', 'Fog')

df_in.set_value( $i$, 'event', 'fog/mist')

elif smoke != -1 :

df_in.set_value ( $i$, 'obscure', 'Smoke')

df_in.set_value( $i$, 'event', 'smoke')

elif SA $!=-1$ :

df_in.set_value( $i$, 'obscure', 'Sandstorm')

df_in.set_value( $i$, 'event', 'dust/sand obs')

elif mist $!=-1$ :

df_in.set_value( $i$, 'obscure', 'Mist')

df_in.set_value(i, 'event', 'fog/mist')

\# The dust storms code (DS) looks the same as other codes when searched for this way. Check \#the location of DS and the things around it to see if it is actually a

\#dust storm or just a part of another code statement.

elif DS $!=-1$ :

if $D S<$ RMK:

\# DS is a part of DSNT - -> ignore!

df_in.set_value( $i$, 'obscure', 'Duststorm')

df_in.set_value( $i$, 'event', 'dust/sand obs')

\# Now look for info on thunderstorms and lightning

if tstorm != 1 :

df_in.set_value( $i$, 'Tstorm', 'Thunderstorm 0-10 miles')

elif zap $!=-1$ and tstorm $==-1$ :

df_in.set_value(i, 'Tstorm', 'Thunderstorm 10-30 miles')

\# Look for a remark about a passing front

if fropa ! = -1:

if tstorm $==-1$ and zap $==-1$ :

df_in.set_value(i,'Tstorm', 'Frontal Passage no Tstorm')

else:

df_in.set_value(i,'Tstorm', 'Frontal Passage with Tstorm')

\# get the 24 hour precipitation total

if $d p !=-1$ :

if $\mathrm{dp}>\mathrm{RMK}$ :

\# Check to make sure it is in the remarks section

$a=\operatorname{metar}[\mathrm{dp}+2: \mathrm{dp}+6]$

\# Grab the daily precipitation data in 1/100th of an inch

if is_number(a) == True:

\# Make sure you have 4 numbers in the string

$b=$ float $(a)$

$c=b / 100$

df_in.set_value(i, 'dailypre', c)

\# Define new column "event_code" to make it easy to sort the data based on observations of vision, dust, fog, etc.

\# Code for different combinations of low visibility (< 7 nautical miles) and obscurations

$\# \odot=$ no obscurations and high visibility

\# $1.0=$ low visibility, no obscuration reported

\# 2.0 = low visibility and BLDU, DS, SS, PO

\# 2.1 = high visibility and BLDU, DS, SS, PO

\# $3.0=$ low visibility and haze

\# 3.1 = high visibility and haze 
$\# 8.0=$ low visibility and fog $/ \mathrm{mist}$
$\# 8.1=$ high visibility and fog $/ \mathrm{mist}$
$\# 9.0=$ low visibility and smoke
$\# 9.1=$ high visibility and smoke

for $i$ in $\operatorname{range}(\odot$, len $(d f$ in.index $))$ : event_str $=d f \_i n . l o c\left[i\right.$, 'event $\left.^{\prime}\right]$ vis $=d f \_i n . l o c[i$, 'vis_check' $]$

if vis > 7.0:

if event_str == 'none':

df_in.set_value( $i$, 'event_code', $\odot . \odot)$

elif event_str == 'dust/sand obs':

df_in.set_value( $i$, 'event_code', 2.1)

elif event_str == 'haze':

df_in.set_value(i, 'event_code', 3.1)

elif event_str $==$ 'fog/mist':

df_in.set_value( $i$, 'event_code', 8.1)

elif event_str == 'smoke':

df_in.set_value( $i$, 'event_code', 9.1)

elif vis $<=7.0$ :

if event_str == 'none':

df_in.set_value( $i$, 'event_code', 1.0)

elif event_str == 'dust/sand obs':

df_in.set_value( $i$, 'event_code', 2.๑)

elif event_str == 'haze':

df_in.set_value(i, 'event_code', 3.0)

elif event_str $==$ ' $\mathrm{fog} / \mathrm{mist}$ ':

df_in.set_value( $i$, 'event_code', 8.0)

elif event_str == 'smoke':

df_in.set_value(i, 'event_code', 9.0)

print ' '

print 'Data Processed --> Saving File'

print ''

\# Clean Up Processing - A search for mistaken obscurations

for $i$ in range $\left(\odot\right.$, len $\left.\left(d f \_i n . i n d e x\right)\right)$ :

\#index $=\mathrm{df} \_$in.index $[i]$

\# Create an index

metar = df_in.loc $[i$, metar']

\# Get row $i$ of METAR

$\mathrm{PO}=$ metar.find $($ 'PO')

\# Look for Dust Devil reports

POINT = metar.find ('POINT')

\# Look for PO occurring as part of DEWPOINT

PORAI = metar. find ('PORAI')

\# Look for PO occurring as part of TEMPORARIRILY

if PO $!=-1$ :

\# Scan for Po being part of a different report, if so, set event_code to zero

if $\mathrm{PO}==$ POINT:

df_in.set_value $(i$, 'event_code', $\odot . \odot)$

df_in.set_value( $i$, 'event', 'station down')

print metar

elif PO == PORAI:

df_in.set_value(i, 'event_code', $\odot . \odot)$

df_in.set_value( $i$, 'event', 'station down')

print metar

\# OUTPUT Individual Station File - .

df_station = df_in

$\mathrm{df}$ _station $=\mathrm{df}$ _station.drop $(\mathrm{df}$ _station.columns $[[13,14,15,16,17,18,19,20]]$, axis=1)

\# Remove the unwanted/unnecessary columns from the data

output_station_file $=$ current_file $[:-4]+{ }^{\prime} \_$PROCESSED.txt '

\# generate output file name 


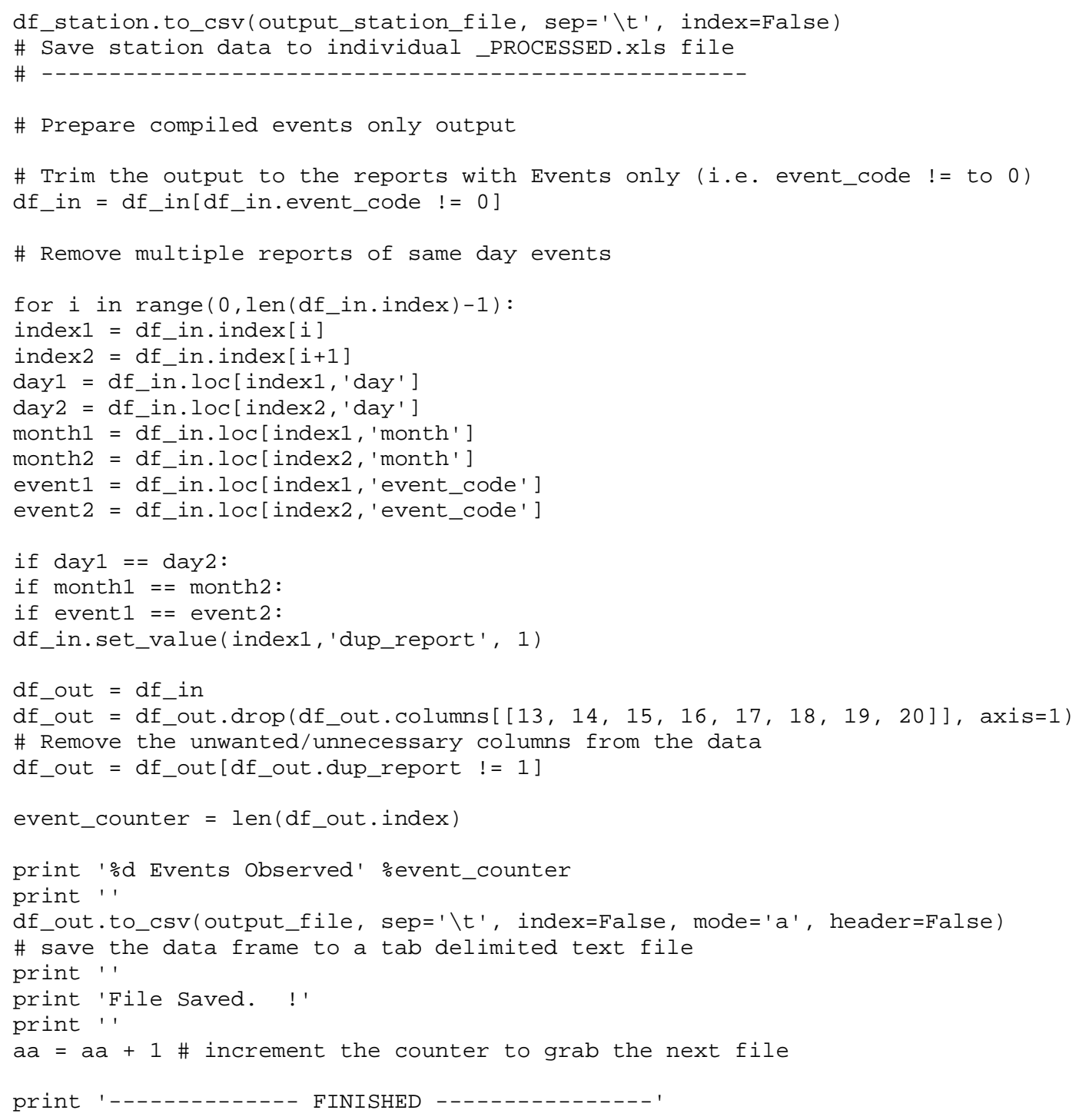




\section{Appendix B: Creating MODIS Imagery}

To download MODIS imagery, create a folder that contains the Python script entitled “MODIS_Dust_Detection.py" (included below). All downloaded images will automatically save to this folder. Type the following command: python MODIs_Dust_Detection.py. A prompt will appear asking for the southern latitude, northern latitude, western longitude, and eastern longitude boundaries of the area of interest. Note, a negative latitude refers to the Southern Hemisphere while a negative longitude refers to the Western Hemisphere. A second prompt will ask for a start and end date for which the satellite imagery will be downloaded. Using the list of dates generated through inspecting the ASOS weather station data, enter a date (in yyyy $/ \mathrm{mm} / \mathrm{dd}$ format) associated with a single dust event. Downloading MODIS images can be time consuming, so users should not download images for a period greater than three days at one time. Instead, break up longer multiday dust events into two separate downloads. Finally, a prompt to create an output file name will be displayed. A single day may contain several MODIS images, so files should be named according to the dates they are associated with. An example of user input is shown below.

Python Script Prompts

Enter Southern Latitude Bound

Enter Northern Latitude Bound

Enter Western Latitude Bound

Enter Eastern Latitude Bound

Enter Beginning Date (e.g. 2003/ 12/ 31)

Enter Ending Date (e.g. 2003/ 12/31)

Enter Name for Output File
Example User Inputs

30

40

$-120$

$-105$

$2016 / 02 / 01$

$2016 / 02 / 03$

February_1-3_2016

There are two known processing issues that exist when downloading MODIS imagery. The first involves an error message that states that the download has "Timed Out," which is likely a result of downloading images for a date range that is too long. When this error occurs, split any timed out multiday events into two separate downloads. Alternatively, if a singleday event times out, try downloading it a second time. A second error message, "Too Many Users," is a result of too many individuals downloading MODIS data from NASA (National Aeronautics and Space Administration) at one time. This error message is rare; but when encountered, users should try to redownload the images later. 


\section{MODIS Dust Detection Python Script}

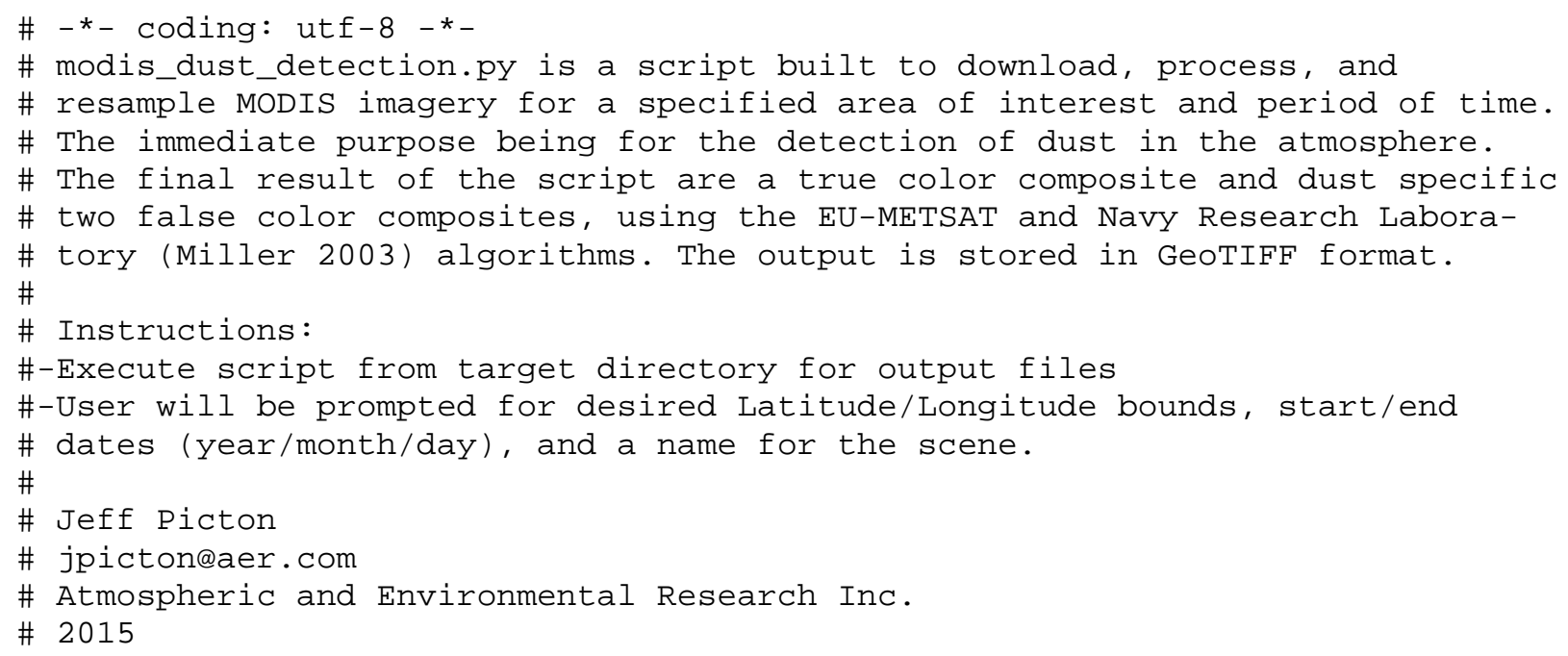


lat2 = raw_input ('Enter Northern Latitude bound: ')

\#lat2 = '40'

lon1 = raw_input('Enter Western Longitude bound: ')

\#lon1 = '-120'

lon2 = raw_input('Enter Eastern Longitude bound: ' )

\#lon2 = '-105'

date1 = raw_input ('Enter beginning date (e.g. 2003/12/31): ')

date2 = raw_input ('Enter ending date (e.g. 2003/12/31): ')

aoi = raw_input('Enter name for output files: ')

\# save imagery info to log file

$\mathrm{ff}=$ open('imagery_log.txt', 'ab')

\# open existing file to append or create a new file

ff.write(lat1+'\t') \# write the data and end with a tab

ff.write (lat2+' $\left.\backslash t^{\prime}\right)$

ff.write (lon $1+$ ' $\backslash \mathrm{t}$ ')

ff.write(lon2+' $\backslash t$ ')

ff.write (date1+' $\left.\backslash t^{\prime}\right)$

ff.write(date2+'ไt')

ff.write(aoi+'\n')

ff.close() \# close the file

\# convert to correct data types

try:

latbnds $=[$ float $($ lat 1$)$, float $($ lat 2$)]$

lonbnds $=[$ float $(\operatorname{lon} 1)$, float $($ lon 2$)]$

date1 = datetime.datetime.strptime $\left(\right.$ date1, $\left.{ }^{\prime} \% \mathrm{Y} / \% \mathrm{~m} / \% \mathrm{~d}^{\prime}\right)$

date2 = datetime.datetime.strptime(date2, $\left.{ }^{\prime} \% \mathrm{Y} / \% \mathrm{~m} / \% \mathrm{~d}^{\prime}\right)$

except :

print('Invalid input found - Please try again')

sys.exit()

if latbnds[0]>latbnds[1]:

print('Upper Latitude bound must be greater than lower ') sys.exit( )

if latbnds [0]>latbnds[1]:

print('Upper Longitude bound must be greater than lower') sys.exit()

if date1>date2:

print('End date cannot be before start date')

return latbnds, lonbnds, date1, date2, aoi

\# return list of granules that cover aoi

def findgranules (latbnds, lonbnds, date1, date2):

\# enumerate dates

days $=[$ date $1+$ datetime.timedelta( $n)$ for $n$ in range ((date2-

date1) $\cdot$ days +1$)]$

\# define platform variables

\# -...- Modified because TERRA satellite in safe mode starting 2/19/2016

TERRA_down_date $=$ '2016/02/19'

TERRA_down_date $=$ datetime.datetime.strptime (TERRA_down_date, ' $\% \mathrm{Y} / \% \mathrm{~m} / \% \mathrm{~d}$ ')

AQUA_start_date $=$ '2002/08/13'

AQUA_start_date $=$ datetime.datetime.strptime (AQUA_start_date, ${ }^{\prime} \% \mathrm{Y} / \% \mathrm{~m} / \% \mathrm{~d}$ ')

test_TERRA $=($ date $1-$ TERRA_down_date $) \cdot$ days

test_AQUA $=($ date1-AQUA_start_date $) \cdot$ days 


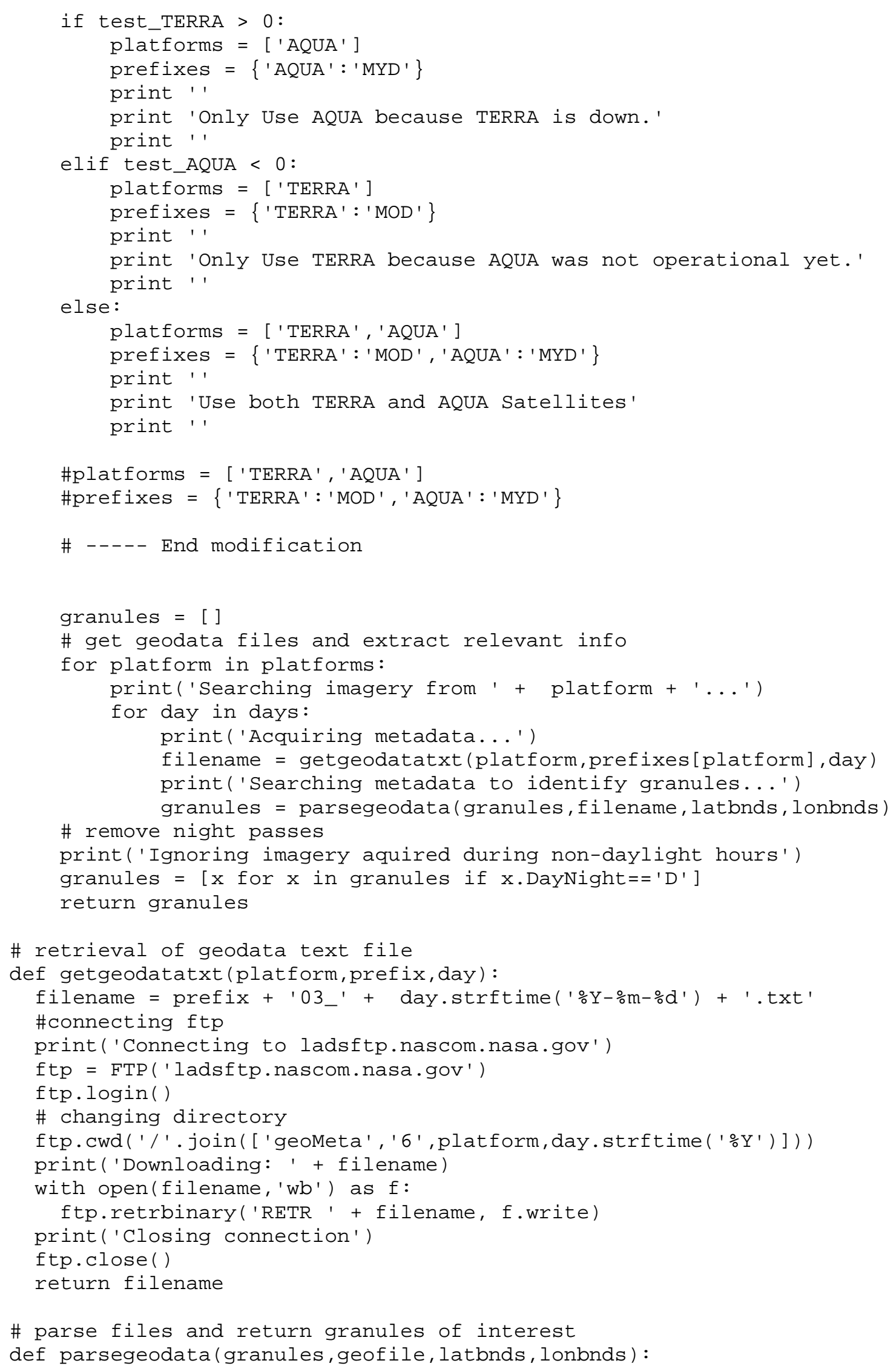




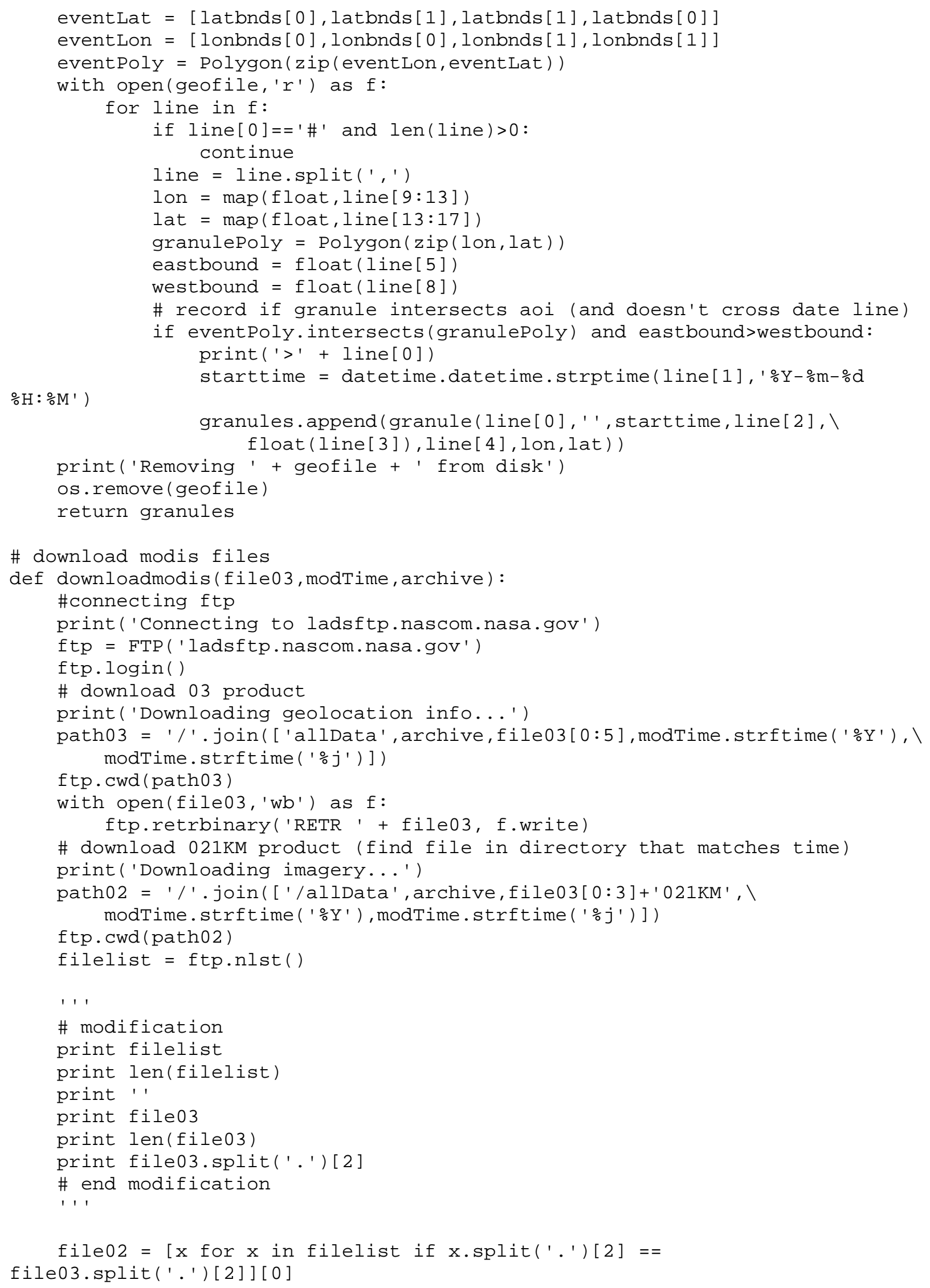


with open(fileo2, ' $w b$ ') as $f$ :

ftp.retrbinary('RETR ' + fileo2, f.write)

print ('Disconnecting')

ftp.close()

return fileo2

\# set flags to appropriate values or nan

def removeflags $(A)$ :

$A=A$.astype ('float64')

$A[A==65533]=32767$ \#saturated

$A[A==65532]=0$ \#zero point

$A[A==65530]=0$ \#below valid range

$A[A==65529]=32787$ \#above valid range

$A[A>32787]=n p$. nan $\#$ all other flags

return $A$

\# convert radiance to brightness temperatures

def $\operatorname{rad} 2 \mathrm{tb}$ ( $\mathrm{rad}$, wavelength):

planck $=6.626176 \mathrm{E}-34 \# \mathrm{~J} \mathrm{sec}$

boltz $=1.380662 \mathrm{E}-23 \# \mathrm{~J} / \mathrm{K}$

cLight $=2.99792458 \mathrm{E}+8 \# \mathrm{~m} / \mathrm{sec}$

$\mathrm{c} 1=2$ * planck * cLight**2

$\mathrm{c} 2=$ planck * cLight / boltz

$\mathrm{V}=1 /$ wavelength $\# 1 / \mathrm{m}$

$\mathrm{R}=\mathrm{rad}^{*} 1 \mathrm{e} 6$ \# $1 \mathrm{e} 6 \mathrm{micron} / \mathrm{m}$

$\mathrm{TB}=\mathrm{c} 2{ }^{*} \mathrm{~V} / \mathrm{np} \cdot \log \left(\mathrm{c} 1^{*} \mathrm{~V}^{*}{ }^{*} 5 / \mathrm{R}+1.0\right)$

return $T B$

\# scale data gamma correction

def scaledata(vals, minin, maxin, minout, maxout, gamma):

vals = vals - minin

vals $=$ vals $/($ maxin-minin $)$

$\operatorname{vals}[\mathrm{np}$. isnan $(\operatorname{vals})]=0$

vals $[$ vals $<0]=0$

$\operatorname{vals}[$ vals $>1]=1$

if not gamma $==1.0$ :

vals $=$ vals ${ }^{*}$ gamma

if not (minout $==\odot$ and maxout $==1$ ):

vals $=$ vals* $($ maxout - minout $)+$ minout

return vals

\#

def readdata(hdfDataFile, hdfGeoFile, latbnds, lonbnds, dlon, dlat):

\# read lat/lon grid

print('Reading geolocation data...')

hdfGeo = SD.SD (hdfGeoFile)

sds $=$ hdfGeo. select ('Longitude')

lon $=$ sds.get ()

sds $=$ hdfGeo.select ('Latitude')

lat $=$ sds.get ()

\# read land/sea mask

print('Reading land/sea mask..')

sds = hdfGeo. $\operatorname{select}($ 'Land/SeaMask')

landSeaMask $=$ sds.get ()

\# read data - emissive 


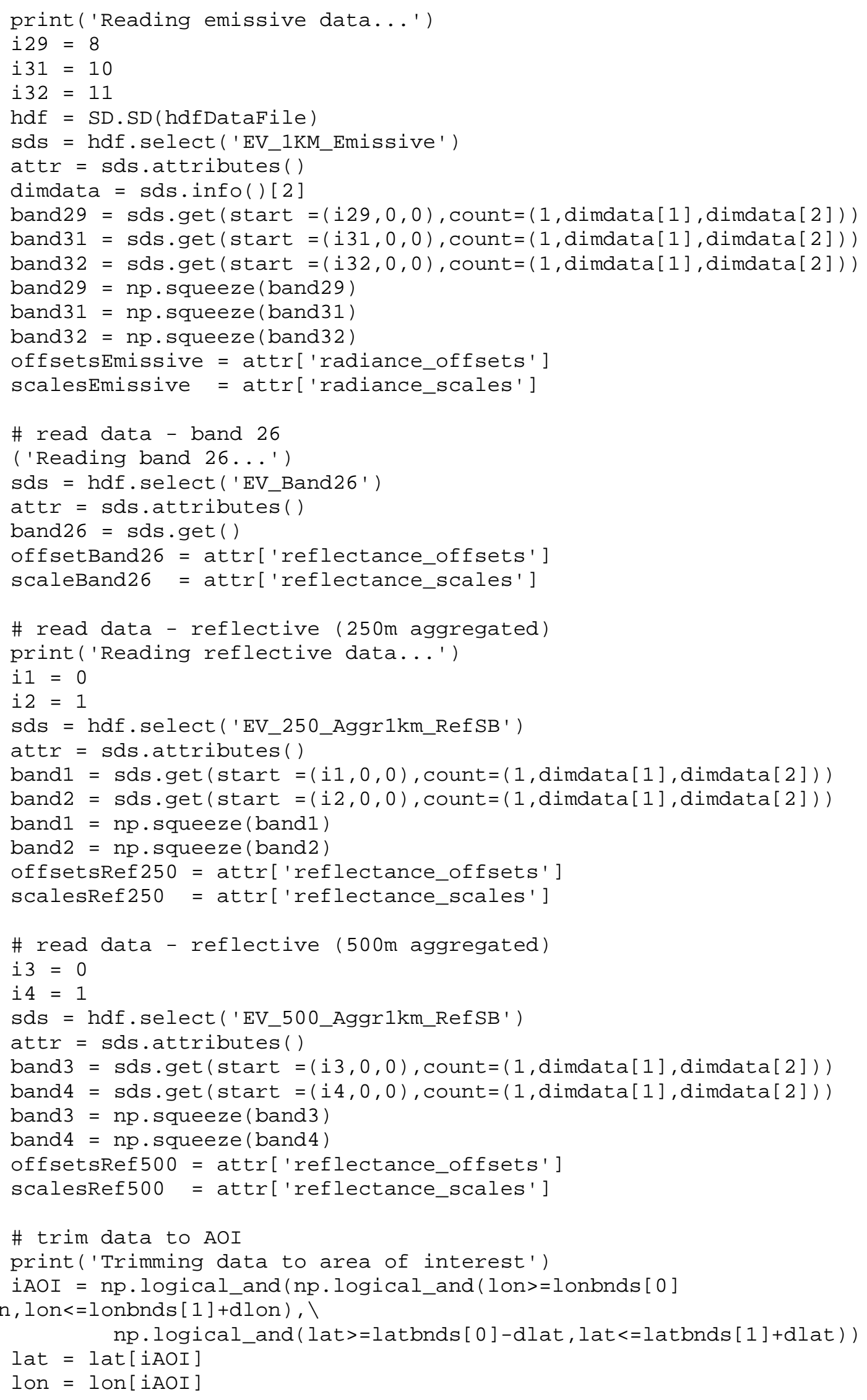




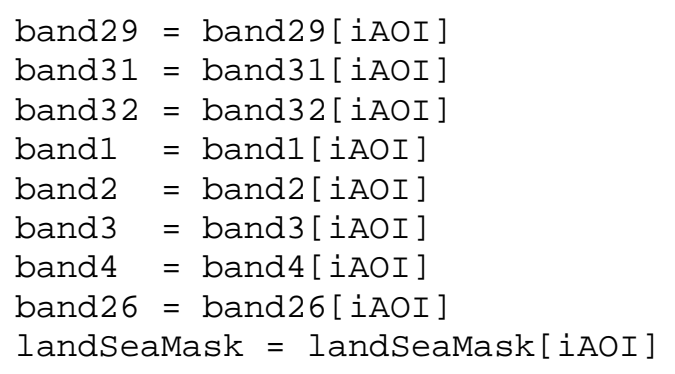

return lon, lat, landSeaMask, band1, band2, band3, band4, band26, band29, band31, band32, offsetsEmissive, scalesEmissive, offsetsRef250, scalesRef250, 1 offsetsRef500, scalesRef500, offsetBand26, scaleBand26, $\backslash$ $i 1, i 2, i 3, i 4, i 29, i 31, i 32$

\# convert data from digital numbers to physical values of interest def convertdata (band1, band2, band3, band4, band26, band29, band31, band32, 1 offsetsEmissive, scalesEmissive, offsetsRef250, scalesRef250, $\mid$ offsetsRef500, scalesRef500, offsetBand26, scaleBand26, I $i 1, i 2, i 3, i 4, i 29, i 31, i 32)$ :

\# set missing values to min/max as appropriate or mark as nan print('Cleaning up missing values')

band29 = removeflags $($ band29)

band31 $=$ removeflags $($ band31)

band32 $=$ removeflags $($ band32)

band1 $=$ removeflags (band1)

band2 = removeflags $($ band 2$)$

band3 = removeflags $($ band 3 )

band $4=$ removeflags $($ band 4$)$

band26 $=$ removeflags $($ band 26$)$

\# convert data to radiance/reflectance

print('Converting digital numbers to radiance/reflectance')

band29 $=($ band29-offsetsEmissive [i29] $){ }^{*}$ scalesEmissive [i29]

band31 $=($ band31- offsetsEmissive [i31] $){ }^{*}$ scalesEmissive [i31]

band32 $=($ band32- offsetsEmissive [i32] $) *$ scalesEmissive [i32]

band1 $=\left(\right.$ band1-offsetsRef250[i1] ${ }^{*}$ scalesRef250[i1]

band2 $=(\text { band2 }- \text { offsetsRef250[i2] })^{*}$ scalesRef250[i2]

band3 $=(\text { band3 }- \text { offsetsRef500[i3] })^{*}$ scalesRef500[i3]

band $4=(\text { band } 4 \text {-offsetsRef500 [i4] })^{*}$ scalesRef500 [i4]

band $26=($ band $26-$ offsetBand 26$){ }^{*}$ scaleBand 26

\# convert emissive data to brightness temperatures

print('Converting radiance to brightness temperature for emissive bands') band29 $=$ rad2tb (band29, 8.55E-6)

band31 $=\operatorname{rad} 2 \mathrm{tb}($ band $31,11.03 \mathrm{E}-6)$

band32 $=\operatorname{rad} 2 \mathrm{tb}($ band $32,12.02 \mathrm{E}-6)$

return band1, band 2 , band3, band4, band 26 , band29, band31, band 32

def readandpreprocessdata(hdfDataFile, hdfGeoFile, latbnds, lonbnds, dlon, dlat):

lon, lat, landSeaMask, band1, band2, band3, band4, band26, band29, band31, band32, I offsetsEmissive, scalesEmissive, offsetsRef250, scalesRef250, 1 offsetsRef500, scalesRef500, offsetBand26, scaleBand26, 1 $i 1, i 2, i 3, i 4, i 29, i 31, i 32=1$ readdata(hdfDataFile, hdfGeoFile, latbnds, lonbnds, dlon, dlat) 
band1, band2, band3, band4, band26, band29, band31, band32 =

convertdata (band1, band2, band 3 , band4, band26, band29, band31, band32, 1 offsetsEmissive, scalesEmissive, offsetsRef250, scalesRef250, I

offsetsRef500, scalesRef500, offsetBand26, scaleBand26, I $i 1, i 2, i 3, i 4, i 29, i 31, i 32$ )

return lon, lat, band1, band2, band3, band4, band26, band29, band31, band32, landSeaMask

\# write data to geotiff

def writeGeoTIFF(R, G, B, lonbnds, latbnds, dlon, dlat, outfile):

\# write data to geotiff

driver = gdal.GetDriverByName('GTiff')

gtiff = driver.Create (outfile,R.shape[0],R.shape[1],3,gdal.GDT_Byte)

gtiff.GetRasterBand(1).WriteArray (np.transpose (R))

gtiff.GetRasterBand (2).WriteArray (np.transpose (G))

gtiff.GetRasterBand (3).WriteArray (np.transpose(B))

\# set projection information

gtiff.SetGeoTransform([lonbnds[ $\odot]$, dlon, $\odot$, latbnds [1], $\odot,-$ dlat $]$ )

srs = osr. SpatialReference ()

srs. SetWellKnownGeogCS( 'WGS84' )

gtiff.SetProjection(srs.ExportToWkt ( ))

\# true color composite

def truecolor (band1, band3, band4):

$\operatorname{Rmin}=0.1$

$\operatorname{Rmax}=0.67$

Rgamma $=0.5$

$\mathrm{R}=$ scaledata (band1, Rmin, Rmax, $\odot, 255$, Rgamma)

Gmin $=0.1$

Gmax $=0.67$

Ggamma $=0.5$

$\mathrm{G}=$ scaledata (band4, Gmin, Gmax, 0,255 , Ggamma)

Bmin $=0.1$

$\operatorname{Bmax}=0.67$

Bgamma $=0.5$

$\mathrm{B}=$ scaledata (band3, Bmin, Bmax, $\odot, 255$, Bgamma)

return $R, G, B$

\# EU-METSAT False color algorithm

def falsecolorEUMETSAT (band29, band31, band32) :

$\operatorname{Rmin}=-4$

$\operatorname{Rmax}=2$

$\mathrm{R}=$ scaledata (band32-band31, Rmin, $\operatorname{Rmax}, 0,255,1.0$ )

Gmin $=0$

$\operatorname{Gmax}=15$

Ggamma $=2.5$

$\mathrm{G}=$ scaledata (band31-band29, Gmin, Gmax, $\odot, 255$, Ggamma)

$\operatorname{Bmin}=-12$

Bmax $=16$

$B=$ scaledata (band31-273.15, Bmin, Bmax, $0,255,1.0$ )

return $R, G, B$

\# NRL False color algorithm (Miller, 2003; GRL)

def falsecolorNRL (band1, band2, band3, band4, band26, band31, band32, landSeaMask): 


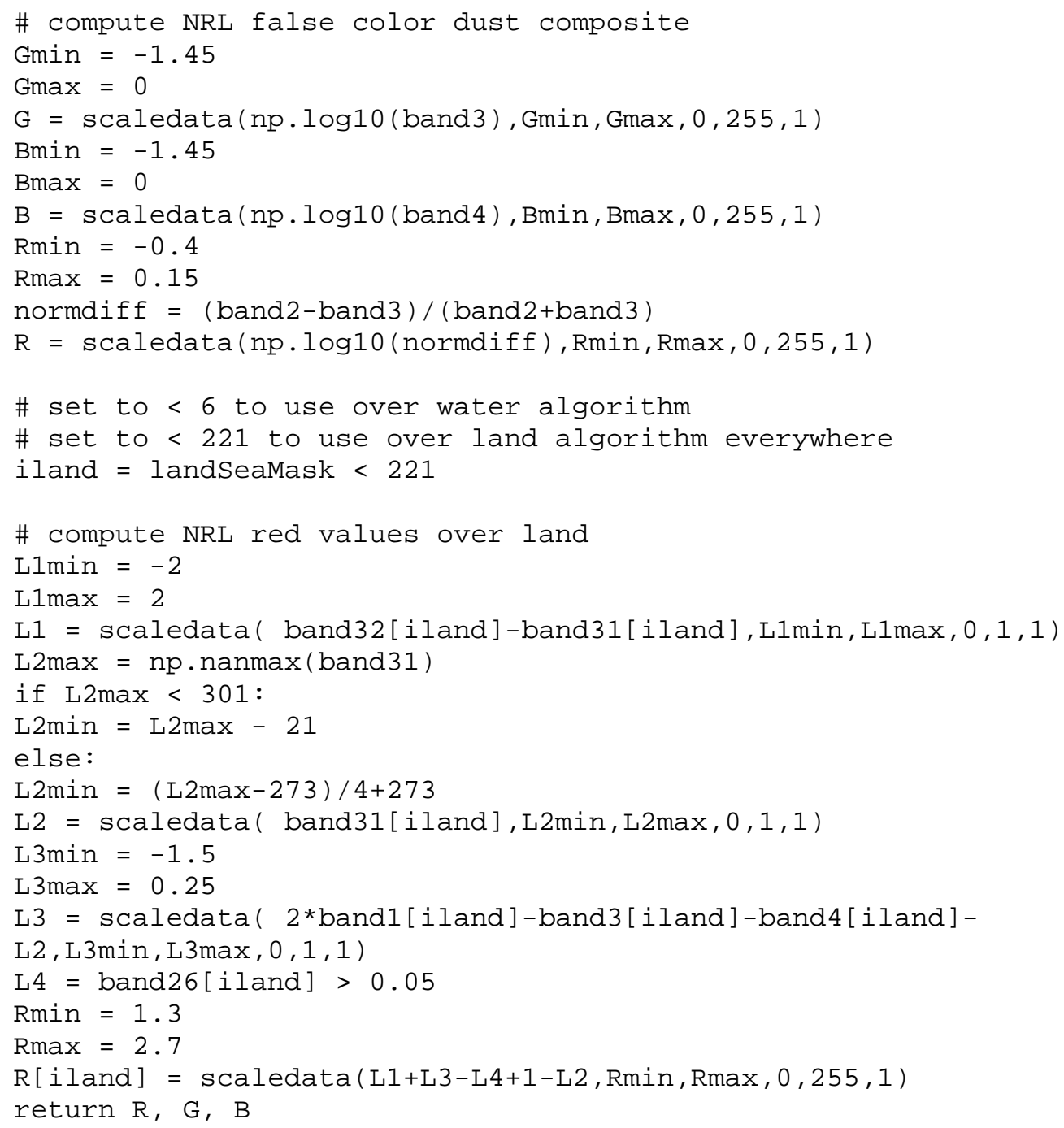

loni, lat $i$, band $1 i$, band $2 i$, band $3 i$, band $4 i$, band26 $i$, band29 $i$, band $31 i$, band $32 i$, landSea Maski $=1$ 


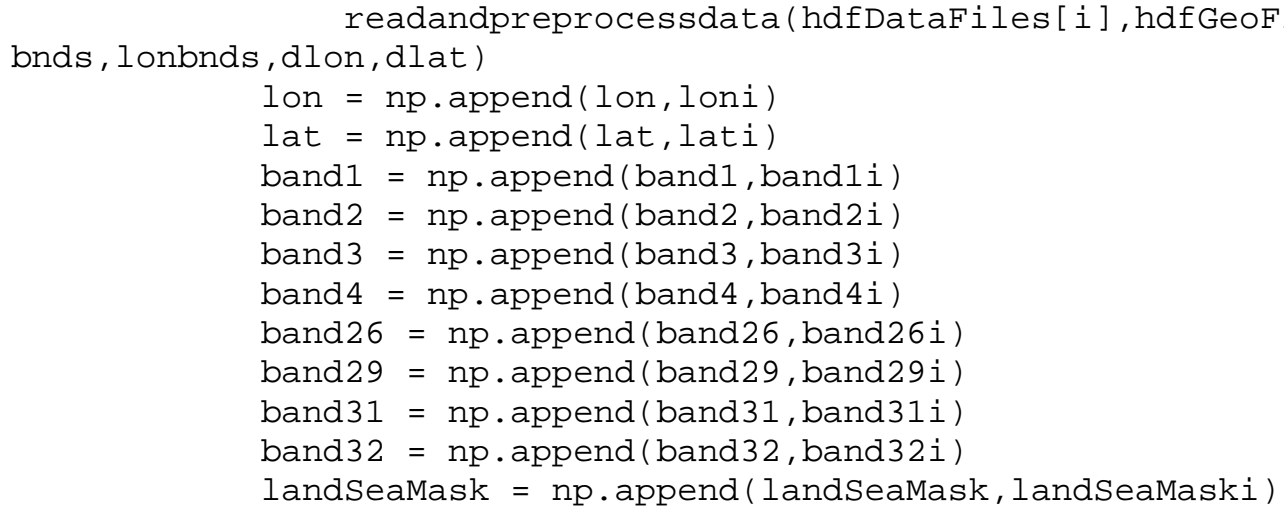

\#delete hdf files

print('Removing raw imagery and geolocation data from disk')

for $f$ in hdfDataFiles:

os. remove $(f)$

for $f$ in hdfGeoFiles:

os. remove ( $f)$

\# true color image

print('Creating true color composite')

Rtrue, Gtrue, Btrue = truecolor (band1, band3, band4)

\# compute EU-METSAT false color dust composite

print('Creating EU-METSAT false color dust composite')

Reumetsat, Geumetsat, Beumetsat = falsecolorEUMETSAT (band29, band31, band32)

\# compute NRL false color dust composite

print('Creating NRL false color dust composite')

Rnrl, Gnrl, Bnrl = I Mask)

falsecolorNRL (band1, band2, band3, band4, band26, band31, band32, landSea-

\# reshape/concat lat/lon data for interpolation (not a regular grid)

latlon $=n p$. concatenate $(($ lon. reshape $((-1,1))$, lat. reshape $((-1,1)))$, axis $=1)$

\# output coordinates

longrid, latgrid = np.mgrid[lonbnds[0]:lonbnds[1]:dlon, latbnds[1]:lat bnds [०]:-dlat]

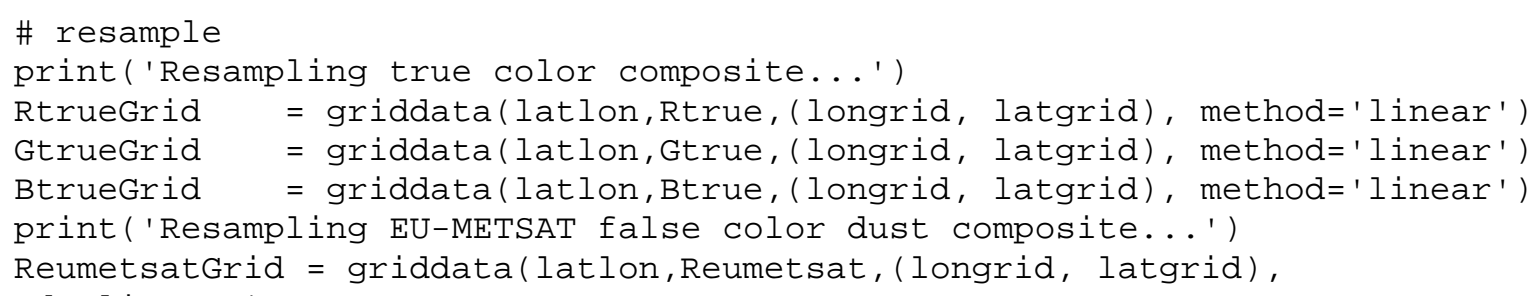




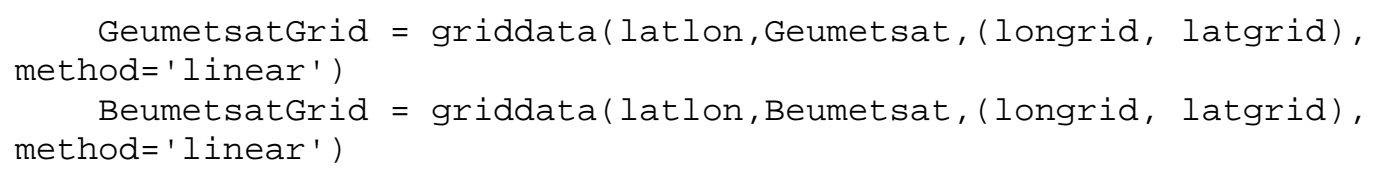

writeGeoTIFF(RnrlGrid, GnrlGrid, BnrlGrid, । filebase, 'NRL', 'tif'])) lonbnds, latbnds, dlon, dlat, ' . ' . join( [out -

writeGeoTIFF(Rtruegrid, GtrueGrid, BtrueGrid, । or', 'tif'])) lonbnds, latbnds, dlon, dlat, ' . ' . join( [outfilebase, 'Truecol-

\# create geotiffs

def processdata(granules, latbnds, lonbnds, aoi):

\# (granules from the same platform during the same orbit are combined) geofiles $=$ [granules [0].File03]

dataFiles $=[$ granules $[0]$. File 02$]$

startTime $=$ granules [0].StartTime

searchTime $=$ granules $[0]$. StartTime

for $i$ in range $(1$, len(granules)):

searchTime $=$ searchTime + datetime.timedelta(minutes=5)

\# if next granule is in continuous set, add on

if granules[i]. StartTime $==$ searchTime:

geoFiles .append (granules [i] . File03)

dataFiles.append (granules [i] . File@2)

\# otherwise, create geotiffs and start new set else:

if $\operatorname{len}($ geofiles $)==1$ :

print('Processing granule ' $+\operatorname{str}(i)+$ ' of ' I else: $+\operatorname{str}(\operatorname{len}($ granules $))+' \ldots ')$

print('Processing granules ' + str(i-len(geoFiles)+1) +1 ' through ' + str(i) + 'of' + str(len(granules)) + '...')

Time, aoi) createfalsecolor(latbnds, lonbnds, geoFiles, dataFiles, start-

$$
\begin{aligned}
\text { geoFiles } & =\text { granules }[i] . \text { File@3] } \\
\text { dataFiles } & =[\text { granules }[i] . \text { File@2] } \\
\text { startTime } & =\text { granules }[i] . \text { StartTime }
\end{aligned}
$$




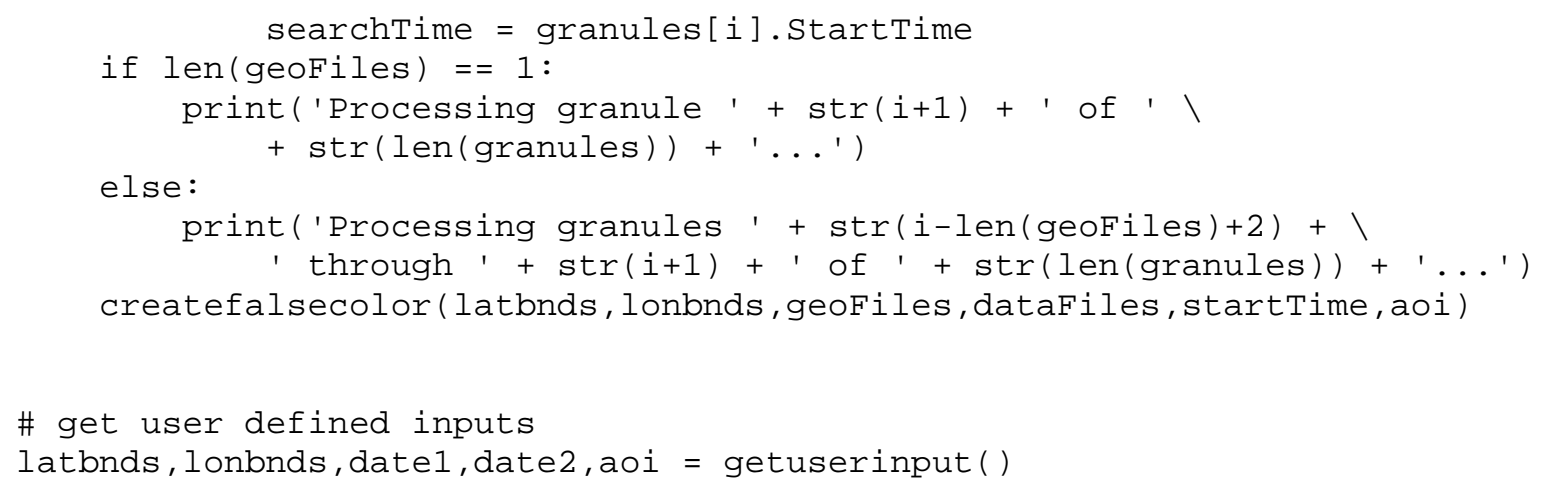




\section{Appendix C: ArcMap Mapping Procedures}

Create a folder entitled "Plume_Head_Mapping"; and within it, create "MODIS Imagery" and "Shapefiles" folders. Place all MODIS imagery deemed suitable for mapping into the MODIS Imagery folder. Open ArcMap and select the "Blank Map" template to open a new project file. To begin building the project file, add a basemap by clicking on the downward facing arrow on the right side of the "Add Data" button (Figure C-1). From the drop down menu, select "Add Basemap" and select "Imagery." Highresolution $(1 \mathrm{~m})$ satellite imagery of the globe will load into the project file and will be listed under the Table of Contents on the left-hand side of the screen (see Figure C-1). To add MODIS imagery, click on the Arc Catalog button (see Figure C-1). Right click "Folder Connections," select "Connect to Folder," navigate to the "Plume_Head_Mapping" folder, and press "OK." The "Plume_Head_Mapping" folder will appear under "Folder Connections" and can be expanded by clicking the small plus sign to the left of the folder name. Open the MODIS Imagery folder located within Plume_Head_Mapping, and all of the images within that folder will appear in the Arc Catalog (see Figure C-1). To add an image to the project file, simply click on it in the Arc Catalog and drag it over to the Table of Contents.

Mapping is conducted by creating and editing point shapefiles. Shapefiles are spatially oriented points, lines, or polygons that are representative of other data (e.g., features on a map). To create a new shapefile, open Arc Catalog; and navigate to the Shapefiles folder, right click on it, and select "New," then "Shapefile," and a "Create New Shapefile" window will appear. Enter a name, and select "Point" under "Feature Type." Under "Description," click "Edit," and a "Spatial Reference Properties" window will open. A commonly used coordinate system is WGS 1984, or the World Geodetic System 1984. To navigate to this system, click the "Geographic Coordinate System" folder, click the "World" folder, highlight "WGS 1984," and click "OK." 
Figure C-1. A screenshot of an ArcMap project file. The top panel shows an enlarged version of the top left corner of the project file. Within the top panel, the red ovaldepicts the location of the Add Data button while the blue ova/highlights the location of the Table of Contents. The green ovaldepicts the location of the Arc Catalog, which, when selected, opens a catalog on the right-hand side of the project file. The pink ovalshows the location of the Editor Toolbar. The expanded Arc Catalog is shown in the lowerpanel, and the contents of the MODIS Imagery folder are enlarged.

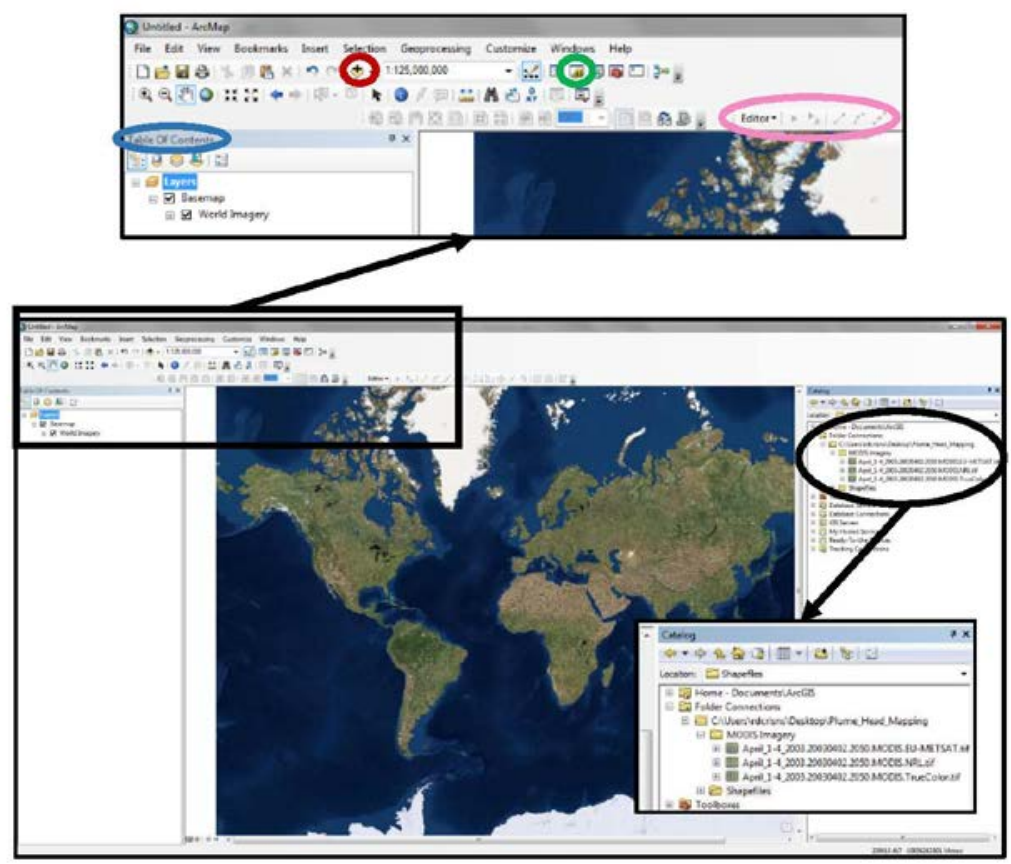

To map plume heads, click "Customize" at the top of the screen, select "Toolbars," and click on "Editor." The Editor Toolbar will appear in the project file (see Figure C-1). In the Editor Toolbar, click "Editor" and then "Start Editing." To the right of the screen, a "Create Features" window will appear. If this window is not visible, click on the pull-down menu on the Editor Toolbar, expand "Editing Windows," and select "Create Features." In the "Create Features" window, click on the name of the shapefile that will be edited to activate the tool that allows users to place new points. Once activated, a new point will be placed on the map anywhere a user clicks. If unwanted points are accidentally placed, remove them by selecting the black arrow in the Editor Toolbar, highlighting the unwanted point, and pressing delete. To save any edits, click "Editor" on the Editor Toolbar, and select "Save Edits." 


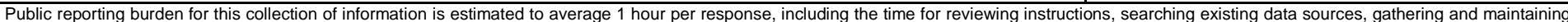

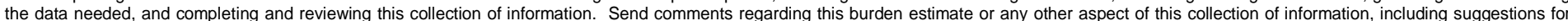

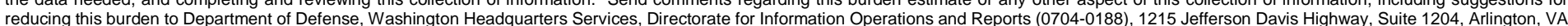

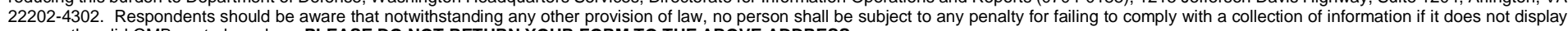
a currently valid OMB control number. PLEASE DO NOT RETURN YOUR FORM TO THE ABOVE ADDRESS.
1. REPORT DATE (DD-MM-YYYY)
2. REPORT TYPE
May 2017
Technical Report/Final

3. DATES COVERED (From - To)

\section{TITLE AND SUBTITLE}

Subjective Mapping of Dust-Emission Sources by Using MODIS Imagery:

Reproducibility Assessment

5a. CONTRACT NUMBER

5b. GRANT NUMBER

5c. PROGRAM ELEMENT NUMBER

\section{AUTHOR(S)}

\section{5d. PROJECT NUMBER}

Samantha N. Sinclair and Sandra L. Jones

5e. TASK NUMBER

ERDC 6.2

5f. WORK UNIT NUMBER

\section{PERFORMING ORGANIZATION NAME(S) AND ADDRESS(ES)}

U.S. Army Engineer Research and Development Center (ERDC)

Cold Regions Research and Engineering Laboratory (CRREL)

8. PERFORMING ORGANIZATION REPORT NUMBER

72 Lyme Road

Hanover, NH 03755-1290

ERDC/CRREL TR-17-8

9. SPONSORING I MONITORING AGENCY NAME(S) AND ADDRESS(ES)

10. SPONSOR/MONITOR'S ACRONYM(S)

Assistant Secretary of the Army for Acquisition, Logistics, and Technology

103 Army Pentagon

Washington, DC 20314-1000

11. SPONSOR/MONITOR'S REPORT NUMBER(S)

\section{DISTRIBUTION / AVAILABILITY STATEMENT}

Approved for public release; distribution is unlimited.

\section{SUPPLEMENTARY NOTES}

ERDC 6.2 Geospatial Research and Engineering (GRE) ARTEMIS STO-R DUST-CLOUD

\section{ABSTRACT}

Dust storms (5 to $100 \mathrm{~km}$ across) often originate from multiple dust-emission sources (1 to $10 \mathrm{~km}$ across). Remote-sensing-based dustsource identification is a challenge. A previous study developed a subjective approach for mapping dust sources by using enhanced MODIS satellite imagery; therefore, this study conducted mapping exercises to assess the reproducibility of this technique amongst multiple analysts and in different regions. Multiple staff members independently analyzed satellite imagery for mappable dust sources for Southwest Asia and the Southwest United States. Mapped points were considered reproducible if the location of dust emission plumes identified by all participants could be constrained to a $10 \mathrm{~km}$ buffer. Using this definition, point-source locations were $28 \%$ reproducible in Southwest Asia and 85\% reproducible in the Southwest United States. Increasing the allotted buffer to $15 \mathrm{~km}$, however, improved results to $71 \%$ and $100 \%$, respectively. Mapped dust sources for Southwest Asia were compared to geomorphic landform maps. At the 1:750,000 map scale, points mapped by all analysts for a single dust plume tended to overlay one landform, while at the 1:100,000 map scale, points were strewn across several landforms. Results suggest that the methodology is reproducible for certain applications but that location-uncertainty tolerance affects perceived conclusions.

\section{SUBJECT TERMS}

Airborne dust, Dust detection, Dust emission, Dust storms, GIS, MODIS, Remote sensing, Remote-sensing images, Reproducibility

16. SECURITY CLASSIFICATION OF:

\section{a. REPORT}

Unclassified

\section{b. ABSTRACT}

Unclassified

\section{c. THIS PAGE}

Unclassified

17. LIMITATION
OF ABSTRACT
SAR

\section{NUMBER} OF PAGES

61

\section{9a. NAME OF RESPONSIBLE} PERSON

19b. TELEPHONE NUMBER (include area code) 